\title{
Geometrical Optics and the Corner Problem
}

\author{
L. Sarason \& J. A. Smoller \\ Communicated by G. STRANG
}

\section{Introduction}

The mixed problem in a half-space for a first-order strictly hyperbolic linear system in $N$ unknowns, namely

$$
L u \equiv u_{t}-\sum_{j=1}^{n} A_{j}(x, t) \partial_{x_{j}} u=f,
$$

has been extensively studied by several authors (see, for example, $[11,6,17,27,32]$ ). By constructing a symmetrizer for the problem, KreIss [17] was able to give necessary and sufficient conditions for the constant coefficient mixed problem to be well-posed in $L_{2}$, as well as sufficient conditions in the case of variable coefficients.

For the corner problem, even for a single higher order equation, much less is known. KUPKA \& OSHER [15] solved such a problem for the wave equation, the results of SARASON $[33,34,35]$ yield sufficient conditions for a class of symmetrizable systems, and OSHER [23] showed how to construct a symmetrizer under certain assumptions.

In this paper we approach the corner problem from the point of view of geometrical optics. (For background in the theory of geometrical optics, see for example, [14], [18] and [20].) Thus we take highly oscillatory data and follow its evolution along bicharacteristic rays, including multiple reflections on the various boundary faces. Assuming the problem to be well-posed, we prove that the geometric optics description yields a good approximation to the true solution, and hence that the associated geometric optics problem is also well-posed.

We study the geometry of the ray history in some detail for strictly hyperbolic polynominals, and we give a method for constructing first order systems whose associated (real) algebraic variety induces certain types of behavior of the ray history. Of particular interest to us are examples of systems with constant coefficients which admit "trapped" or even "periodic" rays. In particular, using such an example, we construct a $4 \times 4$ strictly hyperbolic system in two space variables, whose reduced part is elliptic, with boundary conditions which on each face are correct in the sense of KREISS [17] but such that the mixed problem cannot be well-posed. By use of the results of STRANG [40], it is easy to show that no such example is possible for a $2 \times 2$ strictly hyperbolic system (cf. OsHER [23] for an example of a nonstrictly hyperbolic $2 \times 2$ system). Since the bulk of the paper 
deals with constant coefficients, we postpone until $\S 11$ the technically more complicated variable coefficient case. Our results seem to be related to the work of DUFF [8] and to the recent results of LAX \& NIRENBERG [22] and of DUISTERMAAT \& HöRMANDER [9] on wave-front sets and their reflection at the boundary. We also point out that our geometric optics picture does not include the effect of glancing rays (for the wave equation in the exterior of a star-shaped domain a complete justification has been given by LuDwIG \& MoraweTz [21]; $c f$. also BLOOM [4]), nor do we consider time-asymptotic behavior of the solution, which is of importance in scattering theory, though our methods are related to those of RALSTON [26]. For some additional recent work along these lines see [19], [26].

We now give a brief description of the contents of the paper. In $\$ 2$ we review the geometric optics approximation for the Cauchy problem. In $\$ 3$ we consider the half-space problem with constant coefficients, restricting our attention to those boundary conditions which KREISS [17] proved necessary and sufficient for it to be well-posed, and we define the geometric optics approximation of the mixed problem. We also include a short discussion of symmetric hyperbolic systems. $\$ 4$ is concerned with the corner problem; we prove that if certain a priori estimates hold, then the geometric optics approximation with highly oscillatory data is realistic. In $\S 5$ we reduce the geometric optics problem to one in ray optics; we make assumptions which enable us to avoid considering interactions of rays at the boundary. In $\S 6$ we discuss hyperbolic polynomials and recall some of their geometric properties. In addition, we relate the prolongation of rays to the associated algebraic variety. Trapped rays are discussed in $\S 7$; the main result there is a method for constructing first order strictly hyperbolic systems whose characteristic polynominal has certain properties which allow the existence of a trapped ray. In $\S 8$ we study further properties of trapped rays and give several examples. We show that for $N=2$ there cannot exist trapped rays for systems with elliptic reduced part, and we prove the existence of "limit cycle" periodic trapped rays. $\S 9$ is the major section of our paper. There we demonstrate that trapped rays can destroy the well-posed nature of the problem. In $\S 10$ we discuss some relations between trapped rays and lacunas, but only for the case of two space variables. Finally in $\$ 11$ we extend the geometric optics solution of the mixed problem to the variable coefficient case.

\section{The Initial-Value Problem}

In this section we shall let $L$ denote a strictly hyperbolic $N \times N$ first order linear system of partial differential operators in $n+1$ independent variables, of the form

$$
L u=\partial_{t} u-\sum_{i=1}^{n} A_{i}(x, t) \partial_{x_{i}} u
$$

where each $A_{i}(x, t)$ is a sufficiently smooth $N \times N$ matrix. Then (cf. KELLER [14], LAX [18], LUDWIG [20]) approximate solutions of the equation $L u=0$ can be constructed by an extension of the method of geometric optics. Thus, we consider approximate solutions (depending on a parameter $\lambda$ ) of the form

$$
u_{\lambda}=u_{\lambda}(x, t)=e^{i \lambda \phi(x, t)}\left[a(x, t) r_{1}(x, t)+\lambda^{-1} \sum_{j=2}^{N} b_{j}(x, t) r_{j}(x, t)\right] \text {, }
$$


where $\phi$ is a solution of the eikonal equation

$$
\operatorname{det}\left|\phi_{t}-\sum A_{j} \phi_{x_{j}}\right|=0
$$

and $r_{j}$ are eigenvectors of the matrix $\mathscr{M}(\phi)=\phi_{t}-\sum A_{j} \phi_{x_{j}}$, with $\mathscr{M} r_{1}=0$. The scalar transport coefficients $a$ and $b_{j}$ satisfy appropriate conditions which are determined as follows: Let $\sigma_{j}$ be the eigenvalues of $\mathscr{M}$ with $\sigma_{1}=0$, and let $l_{j} r_{j}=1$, where $l_{j}$ is the corresponding left eigenvector. Substituting the expression (2.2) for $u$ in (2.1), we get (using the summation convention)

$$
e^{-i \lambda \phi} L u_{\lambda}=\left(a_{t}-A_{j} a_{x_{j}}\right) r_{1}+a\left(r_{1, t}-A_{j} r_{1, x}\right)+\sum \sigma_{j} b_{j} r_{j}+O\left(\lambda^{-1}\right) .
$$

Next, we set $L u_{\lambda}=O\left(\lambda^{-1}\right)$ and multiply (2.4) on the left successively by $l_{j}, j=1, \ldots, N$; this gives

$$
a_{t}-\left(l_{1} A_{j} r_{1}\right) a_{x_{j}}+l_{1}\left(r_{1, t}-A_{j} r_{1, x_{j}}\right) a=0
$$

and

$$
b_{j}=-\sigma_{j}^{-1} l_{j}\left[\left(a_{t}-A_{j} a_{x_{j}}\right) r_{1}+a\left(r_{1, t}-A_{j} r_{1, x_{j}}\right)\right]
$$

for $j=2, \ldots, N$. LAX [18] observed that the direction of differentiation in (2.5) is along a null bicharacteristic of $L$. The bicharacteristics of $L$ are defined by the equations

$$
\begin{aligned}
& \dot{x}_{i}=p_{\xi_{i}}, \quad \dot{t}=p_{\tau}, \\
& \dot{\xi}_{i}=-p_{x_{i}}, \quad \dot{\tau}=-p_{t},
\end{aligned}
$$

where $p=p(x, t ; \xi, \tau)$ is the determinant $\operatorname{det}\left(\tau-A_{j} \xi_{j}\right)$, and the null bicharacteristics are those along which $p=0$.

If $p$ is complex, then we want to use the real bicharacteristics of $L$. These are of the form

$$
\begin{aligned}
& \dot{x}_{i}=z p_{\xi_{i}}, \quad \dot{t}=z p_{t}, \\
& \dot{\xi}_{i}=-z p_{x_{i}}, \quad \dot{\tau}=-z p_{t},
\end{aligned}
$$

where $z=z(x, t ; \xi, \tau)$ is a complex number of modulus 1 . (To see this, let $V$ be the real variety defined by $p=0$; then $\nabla_{x, t, \xi, \tau} p=\bar{q}+i \bar{r}$, where $\bar{q}$ and $\bar{r}$ are real. Since both $\bar{q}$ and $\bar{r}$ are normal to $V$, and $V$ is smooth, they must be proportional.)

Now given $\nabla_{x} \phi$, equation (2.3) has $N$ distinct solutions $\phi_{t}$, which we write as

$$
\phi_{t}=q_{i}\left(x, t, \nabla_{x} \phi\right), \quad i=1,2, \ldots, N ;
$$

one of these $q_{i}$ 's, together with initial values $\phi(x, 0)$ for $\phi$, determines $\phi$ along each ray until it hits a caustic (envelope of rays). Once $\phi$ is chosen, $u_{\lambda}$ is determined in the same region by choice of $\lambda$ together with initial values $a(x, 0)$ for $a$.

Our analysis will take place in a small region of space-time. In such a region $\phi$ and $u$ will differ only slightly from solutions of the equation with constant coefficients. (This remark holds for the mixed problem as well, provided we restrict attention to a finite number of reflections and require that these rays avoid tangential (glancing) directions.) We therefore study constant coefficient equations and assume that the $A_{i}$ 's are constant; the variable coefficient case 
requires more sophisticated tools and will be considered in $\S 11$. According to (2.7), then, $\dot{\xi}_{i}=\dot{\tau}=0$ and $r_{t}=r_{x_{i}}=0$. Thus the rays are straight lines along which $a$ is constant and $b_{j} \equiv 0$.

Remark 2.1. We can also solve, approximately, the initial-value problem with $L u=0$ replaced by

$$
L u=e^{i \lambda \phi} f_{1}(x, t) r_{1} .
$$

The approximate solution will differ from the one described above only in that the right-hand side of $(2.5)$ will be $f_{1}(x, t)$ rather than zero.

\section{The Mixed Problem in a Half-Space}

In this section we ask that $e^{i \lambda \phi}$ be a plane wave, i.e., that $\phi=\tau t+\xi \cdot x$, where $p(x, t ; \xi, \tau)=0$.

We consider the mixed problem in the region $\Omega \times[0, T]$, where

$$
\Omega=\left\{x: x_{1}>0,-\infty<x_{i}<\infty, i=2, \ldots, n\right\} .
$$

We denote $\left(x_{2}, \ldots, x_{n}\right)$ by $x^{\prime}$, and the dual variables by $\left(\xi_{1}, \xi^{\prime}\right)$. The boundary condition $P u=g$ should be of the type considered by SARASON [32] or KREISS [17]. In KREISs' formulation, we can assume without loss of generality that $A_{1}$ is symmetric and non-singular, and that the vector $u$ is written as $u=u^{\mathrm{I}}+u^{\mathrm{II}}$ with $u^{\mathrm{I}}$ and $u^{\mathrm{II}}$ the orthogonal projections $P_{ \pm} u$ on the negative and positive eigenspace of $A$, respectively. The homogeneous boundary condition then should have the form $u^{\mathrm{I}}\left(0, x^{\prime}, t\right)=S u^{\mathrm{II}}\left(0, x^{\prime}, t\right), t>0$. Set

$$
M=M(\xi, \tau)=i A_{1}^{-1}\left(\tau-\sum_{i>1} \xi_{i} A_{i}\right)=i A_{1}^{-1}\left(\tau-\xi^{\prime} A^{\prime}\right)
$$

For $\operatorname{Im} \tau<0, M$ can be written in the form

$$
U^{-1}\left[\begin{array}{cc}
M_{11} & M_{12} \\
0 & M_{22}
\end{array}\right] U
$$

where the eigenvalues of $M_{11}$ (respectively, $M_{22}$ ) have negative (respectively, positive) real parts.

If $Q_{ \pm}$are the corresponding projections $U^{-1}\left[\begin{array}{ll}0 & 0 \\ 0 & I\end{array}\right] U$ and $U^{-1}\left[\begin{array}{ll}I & 0 \\ 0 & 0\end{array}\right] U$ on the positive and negative eigenspaces of $M_{1}$ respectively, we demand that the operator $\left(P_{-}-S P_{+}\right) Q_{+}$, taking the range of $Q_{+}$into the range of $P_{-}$, has an inverse uniformly bounded in $(\xi, \tau), \operatorname{Im} \tau<0$; i.e., the unit sphere in the null space of $P$ is bounded away from the outgoing space. In this case we shall say that $P$ satisfies condition $B$.

Under these assumptions, SARASON [32] proved the estimate

$$
\begin{aligned}
\langle\|u\|\rangle_{T}^{2} & \equiv \int_{0}^{T}\|u(t)\|_{\Omega}^{2} d t+\int_{0}^{T}\|u(t)\|_{\partial \Omega}^{2} d t \\
& \leqq C_{T}\left\{\int_{0}^{T}\|L u(t)\|_{\Omega}^{2} d t+\int_{0}^{T}\|P u(t)\|_{\partial \Omega}^{2} d t\right\}
\end{aligned}
$$


provided $u(x, 0) \equiv 0$ and $m=2$. The same estimate for the variable real coefficient case for arbitrary $m$ was proved by KREISs [17], who showed that if (3.1) holds both for the direct and adjoint problems, then $P$ satisfies condition $B$.

RALSTON [25] extended KREISS' results to first-order systems with complex coefficients, and RAUCH [27] and KAJITANI [16] improved (3.1) to the stronger estimate

$$
\begin{aligned}
\|u\|_{T}^{2} & \equiv\langle|u|\rangle_{T}^{2}+\|u(T)\|_{\Omega}^{2} \\
& \leqq C_{T}\left\{\int_{0}^{T}\|L u(t)\|_{\Omega}^{2} d t+\int_{0}^{T}\|P u(t)\|_{\partial \Omega}^{2} d t+\|u(0)\|_{\Omega}^{2}\right\} .
\end{aligned}
$$

A similar result for higher-order single equations was derived by SAKAMOTO [31].

We proceed to construct approximate solutions of the mixed problem. Let $(\bar{\xi}, \bar{\tau}) \neq(0,0)$ be a point on $V$, the characteristic variety of $p$, for which $p_{\bar{\xi}}, p_{\tau}<0$. With $0<\varepsilon \ll 1,0<\lambda, \lambda \gg \varepsilon^{-1}$, and with $a_{+}(x, 0) \in C_{0}^{\infty}\left(R_{+} \times R^{m-1}\right)$, choose

$$
a(x, 0) \equiv a_{\varepsilon}(x, 0)=a_{1}(x / \varepsilon, 0)
$$

as initial values for an approximate solution $u_{\lambda, \varepsilon}$ determined by (2.5) and (2.6). We shall denote $u_{\lambda, \varepsilon}$ by $u_{\lambda}$, and discuss later the significance of $\varepsilon$.

Now $u_{\lambda}$ is supported on those bicharacteristic determined by (2.7) which pass through the support of $a_{\varepsilon}(x, 0)$; it does not necessarily satisfy the boundary condition. We can approximate $u_{\lambda}$ on $\Gamma_{1}=\left\{(x, t): x_{1}=0\right\}$ to within order $\lambda^{-1}$ by the function

$$
\begin{aligned}
h\left(x^{\prime}, t\right) & =\left.e^{i \lambda\left(t \bar{\tau}-x^{\prime} \cdot \xi^{\prime}\right)} a\left(-p_{\mathfrak{\tau}}^{-1} p_{\xi_{1}} t, x_{i}^{\prime}-t p_{\bar{\xi}_{t}}^{\prime}, 0\right)\right|_{(\mathfrak{\tau}, \xi)=(\bar{\tau}, \bar{\xi})} \\
& \equiv e^{i \lambda\left(t \bar{\tau}-x^{\prime} \cdot \bar{\xi}^{\prime}\right)} \alpha\left(x^{\prime}, t\right),
\end{aligned}
$$

which is the leading term for $u_{\lambda}$ in (2.2).

At this point it is convenient to study the exact solution $V_{\lambda}$ of the forward mixed problem associated with zero initial conditions and with boundary condition satisfying condition $B$ :

$$
P V_{\lambda}=-P h
$$

Using the results of KREISS [17], we can write $V_{\lambda}$ in the form

$$
\tilde{V}_{\lambda}\left(x_{1}, \xi^{\prime}, \tau\right)=\sum_{j=1}^{J}\left(1+O\left(x_{1}\right)\right) c_{j}\left(\xi^{\prime}, \tau\right) \tilde{h}\left(\xi^{\prime}, \tau\right) e^{i d_{j}\left(\xi^{\prime}, \tau\right) x_{1}} r_{j}\left(\xi^{\prime}, \tau\right),
$$

where $\sim$ denotes the Fourier transform in $\left(x^{\prime}, t\right)$, where each $c_{j}$ is a (scalar) function which we call the reflection coefficient, and where $d_{j}$ is a root of

$$
p\left(d_{j}, \xi^{\prime}, \tau\right)=0
$$

which is distinct from $\xi_{1}$ and has a non-negative imaginary part, and $r_{j}$ is a normalized null vector of the matrix

Here $\zeta_{j}$ is defined as the vector

$$
E\left(\zeta_{j}\right)=\tau-A_{1} d_{j}-\sum_{i>1} A_{i} \xi_{i}
$$

$$
\zeta_{j}=\left(d_{j}, \xi^{\prime}, \tau\right)=\left(d_{j}\left(\xi^{\prime}, \tau\right), \xi^{\prime}, \tau\right)
$$


We remark that $c_{j}$ and $d_{j}$ are homogeneous functions of degrees zero and one, respectively, in their arguments. From KREISs' results, we now see that $c_{j}$ is locally bounded and continuous, except possibly at those points where $d_{j}$ is a multiple root of (3.6).

It is convenient at this point to consider a still further approximation. Since $\tilde{h}(\xi, \tau)=\tilde{\alpha}\left(\tau-\lambda \bar{\tau}, \xi^{\prime}-\lambda \bar{\xi}^{\prime}\right)$ and $\alpha \in C_{0}^{\infty}$, we may, by taking $\lambda$ sufficiently large, ensure that

$$
\left\|h-h_{M, \lambda}\right\|<\lambda^{-2}
$$

where for arbitrary $M>0, h_{M, \lambda}$ is defined by

$$
\tilde{h}_{M, \lambda}= \begin{cases}\tilde{h}, & |\tau-\lambda \bar{\tau}|^{2}+\left|\xi^{\prime}-\lambda \bar{\xi}^{\prime}\right|^{2}<\lambda^{2} / M \\ 0, & \text { otherwise. }\end{cases}
$$

Thus, replacing $h$ by $h_{M, \lambda}$, we commit a small error, and localize the problem in a small cone in $\xi^{\prime}, \tau$ space.

We now construct approximate solutions of the mixed problem of the form

$$
Z_{\lambda}=\sum_{j} e^{i \lambda\left(d_{j}\left(\xi^{\prime}, \bar{\tau}\right) x_{1}+x^{\prime} \cdot \xi^{\prime}-t \bar{\tau}\right)}\left\{\alpha_{j}(x, t) r_{j}^{+}+\sum_{s} \lambda^{-1} \beta_{j s}(x, t) r_{j s}\right\},
$$

where each $r_{j s}$ is a non-null eigenvector of $E\left(\zeta_{j}\right)$.

Definition. The geometric optics approximation is the sum of the zero order* terms of (3.9) for which $d_{j}$ is real.

To continue with our construction, we assume first that the $d_{j}$ 's are distinct. For $d_{j}$ real, the transport coefficients $\alpha_{j}$ and $\beta_{j}$ are treated exactly as in the approximate solution of the Cauchy problem $(\$ 2)$. If $d_{j}$ is not real, we can in fact set $\beta_{j s} \equiv 0$ and $\alpha_{j}\left(x_{1}, x^{\prime}, t\right)=\alpha_{j}\left(0, x^{\prime}, t\right) \psi\left(x_{1}\right)$, where $\psi \in C_{0}^{\infty}, \psi(x) \equiv 1$ for $x<\frac{1}{2}$, $\psi \equiv 0$ for $x>1$. Then using the rapid decrease of the exponential $e^{i \lambda d_{j} x_{1}}$, we conclude that the resulting term, both before and after we apply the differential operator, is $O\left(\lambda^{-1}\right)$ in $L_{2}\left(\Omega \times[0, T]^{\star \star}\right)$.

We note that in order to get an approximate solution satisfying the differential equation uniformly as $\operatorname{Im} d_{j} \rightarrow 0$ (i.e., as we approach a glancing direction), it does not suffice to choose $\alpha$ 's and $\beta$ 's satisfying the exact transport equations, for in that case they will grow exponentially unless the support of $\tilde{\alpha}_{j}\left(0, \xi^{\prime}, \tau\right)$ is appropriately restricted. This entails an approximation of the type (3.7) and (3.8) with $\lambda \rightarrow \infty$ as $\operatorname{Im} d_{j}(\bar{\xi}, \bar{\tau}) \rightarrow 0$; that is the closer the ray comes to a glancing direction, the larger $M \lambda^{-1}$ has to be in (3.8).

We end this section with a short discussion of symmetric hyperbolic systems. The hyperbolic system (2.1) is called symmetric hyperbolic if the coefficient matrices $A_{j}$ are Hermitian. We consider such an operator in the region $\Omega \times[0, T]$, where $\Omega$ is the half-space $x_{1}>0$. If we assume for simplicity that the coefficients are constant, then

$$
u \cdot L u+L u \cdot u=\frac{\partial}{\partial t}|u|^{2}-\frac{\partial}{\partial x_{1}} u \cdot A_{1} u-\sum_{j=2}^{n} \frac{\partial}{\partial x_{j}} u \cdot A_{j} u
$$

$\star$ With respect to $\lambda$.

* If the $d_{j}$ 's are not distinct or come close, we can get a similar estimate for the solution by treating the associated eigenspaces together as a block (cf. \$11). 
If $u \in C_{0}^{\infty}\left(R^{n+1}\right)$, we can integrate (3.10) over $\Omega$. Using Schwarz's inequality, we obtain

$$
\frac{\partial}{\partial t}\|u\|_{\Omega}^{2} \leqq-\left(u, A_{1} u\right)_{\partial \Omega}+2\|u\|_{\Omega}\|L u\|_{\Omega}
$$

Suppose that $A_{1}$ is nonsingular and that $u$ satisfies a homogeneous boundary condition $u \in N$ along $x_{1}=0$, where $N$ is a linear subspace which is maximal positive definite with respect to the form $u \cdot A u$; that is, for some $c>0$, the inclusion

$$
u \in N \text { implies } u \cdot A u>c(u \cdot u) \text {. }
$$

We shall call such a boundary condition strongly dissipative (or, simply, dissipative, if $c=0$ ). Then (3.11) implies

$$
\frac{\partial}{\partial t}\|u\|_{\Omega}^{2}+c\|u\|_{\partial \Omega}^{2} \leqq 2\|u\|_{\Omega}\|L u\|_{\Omega}
$$

From (3.13), together with a similar inequality for the adjoint (time-reversed) mixed problem with adjoint boundary conditions, it is easily shown ([27], [16]) that if $P$ is a matrix whose null space is $N$, then $(3.1)^{\prime}$ holds together with a corresponding estimate for the adjoint problem. Hence $P$ satisfies condition $B$.

\section{The Corner Problem}

Suppose now that the underlying domain $\Omega$ is a region of the form $\Omega=$ $R_{+}^{m^{\prime}} \times R^{n-m}(n>m)$, and set $\Gamma_{i}=\partial \Omega \cap\left\{x_{i}=0\right\}, 1 \leqq i \leqq m$. Assume that on each $\Gamma_{i}$ the boundary condition is of the form $P_{i} u=0$, with $P_{i}$ chosen such that the corresponding half-space problem is $\left(L^{2}\right)$ well-posed in the sense of KREISS (see $\S 3$ ).

We construct geometric optics approximations as above, with initial conditions of the form

$$
e^{i \lambda(t \bar{\tau}-x \cdot \bar{\xi})} a(x, 0) r_{i}
$$

The geometric optics solution for this Cauchy problem will be assumed to vanish on all of $\partial \Omega$ except for a single $\Gamma_{i}$. We construct (as above) a reflected geometricoptics solution emanating from $\Gamma_{i}$, and assume that any given ray packet hits just a single $\Gamma_{i}$. This procedure can be carried out as long as no ray hits an edge or corner.

Henceforth we shall assume that all solutions of the mixed problem satisfy one of the following two types of a priori estimates $(c f . \S 3)$ : either

$$
\begin{aligned}
\|u\|_{T}^{2} & \equiv \int_{0}^{T}\|u(t)\|_{\Omega}^{2} d t+\sum_{i=1}^{n} \int_{0}^{T}\|u(t)\|_{\Gamma_{i}}^{2} d t+\|u(T)\|_{\Omega}^{2} \\
& \leqq C_{T}\left\{\int_{0}^{T}\|L u(t)\|_{\Omega}^{2} d t+\sum_{i=1}^{n} \int_{0}^{T}\left\|P_{i} u(t)\right\|_{\Gamma_{i}}^{2} d t+\|u(0)\|_{\Omega}^{2}\right\}
\end{aligned}
$$


or, for all $u$ such that $u(0) \equiv 0$,

We then have

$$
\begin{aligned}
\langle|u|\rangle_{T}^{2} & \equiv \int_{0}^{T}\|u(t)\|_{\Omega}^{2} d t+\sum_{i=1}^{n} \int_{0}^{T}\|u(t)\|_{\Gamma_{t}}^{2} d t \\
& \leqq C_{T}\left\{\int_{0}^{T}\|L u(t)\|_{\Omega}^{2} d t+\sum_{i=1}^{n} \int_{0}^{T}\left\|P_{i} u(t)\right\|_{\Gamma_{i}}^{2} d t\right\} .
\end{aligned}
$$

Proposition 4.1. (a) Let $u_{\lambda}$ be the true solution and let $\tilde{u}_{\lambda}$ be the geometric optics solution of the mixed problem for (2.1) in $\Omega$ with homogeneous boundary conditions and right-hand side, and with smooth initial conditions (4.1). Suppose that

(*) For some $T>0$ and some $\delta>0$, no rays carrying non-trivial geometric optics data hit an edge or corner, or take a glancing direction along $\partial \Omega$ for

Then if (4.2) holds, we have

$$
0 \leqq t<T+\delta \text {. }
$$

$$
\int_{0}^{T}\left\|u_{\lambda}(t)-\tilde{u}_{\lambda}(t)\right\|_{\Omega}^{2} d t+\left\|\left(u_{\lambda}-\tilde{u}_{\lambda}\right)(T)\right\|_{\Omega}^{2}=O\left(\lambda^{-\frac{1}{2}}\right) \quad \text { as } \lambda \rightarrow \infty .
$$

(b) Assume that (4.3) holds. Let $u_{\lambda}$ be the true solution and let $\tilde{u}_{\lambda}$ be the geometric optics solution of the mixed problem for (2.9) with zero initial condition and inhomogeneous right-hand-side $e^{i \lambda \phi} f_{1}, f_{1} \in C_{0}^{\infty}\left(R^{n+1}\right)$. Let $\operatorname{supp} f_{1}$ be such that, for some $t_{1}>0$, no ray carrying non-trivial geometric optics data hits $\partial \Omega$ at a point $(x, t), t<3 t_{1}$. If $\left(^{*}\right)$ holds, then we have

$$
\left\langle\left|u_{\lambda}-\tilde{u}_{\lambda}\right|\right\rangle_{T}=O\left(\lambda^{-\frac{1}{2}}\right), \quad \text { as } \lambda \rightarrow+\infty \text {. }
$$

Proof. The initial condition is exactly satisfied by $\tilde{u}_{\lambda}$. By adding a function of size $O\left(\lambda^{-1}\right)$, we can satisfy the equation to within $O\left(\lambda^{-1}\right)$ (cf. (2.2)), and by adding another function of $L_{2}$-norm $O\left(\lambda^{-\frac{1}{2}}\right)$, (the $L_{2}$-norm of $e^{-\lambda x_{1}}$ ), we can satisfy the boundary conditions to order $\lambda^{-1}$. We leave it to the reader to check that, by construction of $\alpha_{j}$, the terms reflected from $\Gamma_{i}$ in (3.9) with $\operatorname{Im} d_{j}>0$ are $O\left(\lambda^{-\frac{1}{2}}\right)$ in $L_{2}\left(\Gamma_{k}\right), k \neq i$. In case (b) there is an apparent complication due to the fact that the correction terms as given do not necessarily vanish for $t \leqq 0$; this defect can be remedied, however, by multiplying them by a fixed function $g(t) \in C_{0}^{\infty}(R)$, where $g(t) \equiv 1, t>2 t_{1}, g(t) \equiv 0$ for $t<t_{1}$. The proposition now follows from (4.2) or (4.3), as required.

Remark. If $m=2$, all $d_{j}$ are real and we can replace $O\left(\lambda^{-\frac{1}{2}}\right)$ by $O\left(\lambda^{-1}\right)$ in the conclusion of the proposition.

Definition 4.2. The geometric optics mixed problem is poorly-posed in the sense of (4.2) (respectively (4.3)) if

$$
\sup _{\tilde{u}_{\lambda}} \sup _{T>0} \frac{\left\|\tilde{u}_{\lambda}(T)\right\|_{\Omega}}{\left\|\tilde{u}_{\lambda}(0)\right\|}=\infty
$$

respectively

$$
\left.\sup _{\tilde{u}_{\lambda}} \sup _{T>0} \frac{\int_{0}^{T}\left\|\tilde{u}_{\lambda}(t)\right\|_{\Omega}^{2} d t}{T\left\|\tilde{u}_{\lambda}(0)\right\|^{2}}=\infty\right)
$$


where $u_{\lambda}$ is a geometric optics solution in $0<T<T+\delta, \delta>0$, of the mixed problem with homogeneous boundary conditions.

As a consequence of Proposition 4.1, we have

Theorem 4.3. If the geometric optics mixed problem is poorly posed in the sense of (4.2) (respectively (4.3)), then for the full problem (4.2) (respectively (4.3)) is not valid.

Remark. Replacing $u_{\lambda}(x, t)$ by $u^{\varepsilon}(x, t)=u_{\lambda}(\varepsilon x, \varepsilon t)$, we see that if $\mathrm{N}_{1}$ (respectively $\mathrm{N}_{2}$ ) should hold, then for any $T>0$ we have sup $\left\|u_{\lambda}(0)\right\|^{-1}\left\|u_{\lambda}(T)\right\|=\infty$ (respectively $\left.\sup _{u_{\lambda}}\left(T\left\|u_{\lambda}(0)\right\|^{2}\right)^{-1} \int_{0}^{T}\left\|u_{\lambda}(t)\right\|_{\Omega}^{2} d t=\infty\right)$. This, together with the results of $\S 11$, allows us to apply Theorem (4.3) to the variable coefficient case.

\section{The Ray Optics Approximation}

We shall now reduce the geometric optics problem to one in ray optics; thus we imagine a single (bicharacteristic) ray originating at $(x, 0)$ and associated with the dual variable $(\xi, \tau)$. Our initial data has the form $\alpha_{0} r_{1}$. With a slight abuse of notation, we let $\alpha_{0}(x, t, \xi, \tau)$ represent either the strength $\alpha_{0}$ of the signal, or the full signal $\alpha_{0} r_{1}$.

Our data propagates along bicharacteristics and undergoes various reflections on the boundary faces, so that to each $t \geqq 0$ there corresponds a vector $v(t)=\left\{\alpha_{i}\left(x_{i}, t, \xi_{i}, \tau_{i}\right)\right\}, i=1, \ldots, K_{t}$. (Here $v(t)$ can be considered as the crosssection of a "tree" which in general branches at each reflection point.)

Suppose a bicharacteristic $(x, t, \xi, \tau)$ carrying data $\alpha=\alpha(x, t, \xi, \tau)$ hits $\Gamma_{k}$ at $\left(x_{0}, t_{0}\right)$. Then each reflected bicharacteristic $\left(x, t, \xi_{(j)}, \tau\right)$ issuing from $\Gamma_{k}$ at $\left(x_{0}, t_{0}\right)$ satisfies $\left(\xi_{(j)}\right)_{i}=\xi_{i}, i \neq k$, and carries outgoing data $R_{j} \alpha$ at $\left(x_{0} t_{0}\right)$. Here the ray optics reflection coefficient $R_{j}$ has the form $\mu_{j}(\xi, \tau, k) c_{j}(\xi, \tau, k)$, where $c_{j}$ is the geometric optics reflection coefficient described above (note that $c_{j}=0$ unless the reflected ray is directed into $\Omega$ ), and where $\mu_{j}$ is chosen to compensate for the fact that the "energy" ( $L_{2}$-norm) carried by a "wave packet" reflected from the boundary depends not only on $c_{j}$ but also on the angles involved.

For example, suppose that an incoming wave packet has initial data $u(x, 0)=e^{i(t+x \cdot \xi)} a(x) r$. Then the "unreflected" geometric optics approximation is $u=e^{i(t \tau+x \cdot \xi)} a\left(x_{i}+p_{\tau}^{-1} p_{\xi_{i}} t\right) r$. If the wave packet hits $\Gamma_{i}$, then the induced map from $\Omega \cap \operatorname{supp}(u(x, 0))$ into $\Gamma_{i}$ is given by

$$
T(x)=-p_{\xi_{i}}^{-1} p_{\tau} x_{i}, \quad X_{j}^{\prime}(x)=x_{j}-p_{\xi_{i}}^{-1} p_{\xi_{j}} x_{i}, \quad i \neq j,
$$

and has Jacobian $\partial\left(X^{\prime}, T\right) / \partial x=\left|p_{\xi_{i}}^{-1} p_{\tau}\right|$. If the reflected ray is associated with the vector $\left(\xi_{(j)}, \tau\right)$, then the induced map from $\Omega \cap \operatorname{supp} u(x, 0)$ into $\Omega$ (restricting attention to time $t$ after reflection) has Jacobian

$$
J\left(\xi, \xi^{(j)}, \tau, i\right)=\left|\frac{\partial X}{\partial x}\right|=\left|\frac{p_{\tau}}{p_{\xi_{i}}}\right|_{\xi, \tau}\left|\frac{p_{\xi_{i}}}{p_{\tau}}\right|_{\xi^{(j)}, \tau}
$$

Since we are concerned with $u(\cdot, t)$ as an element of $L_{2}(\Omega)$, we must take $\mu_{j}=J^{\frac{1}{2}}$. 
In dealing with ray optics approximations, it is simpler to avoid problems caused by the interaction on $\partial \Omega$ of several incoming rays which hit at the same point $(x, t)$. To this end, we make some definitions and assumptions.

Let $W_{\mathscr{R}}=(x, 0, \xi, \tau)$ denote the initial point for an incoming ray $\mathscr{R}$ ("incoming" means that it will hit $\partial \Omega$ at some time $t>0$ ). We shall abuse notation by saying that $(x, t) \in \mathscr{R}$ if $(x, t, \xi, \tau) \in \mathscr{R}$. We require that $(x, t) \in \mathscr{R}$ implies that $t \geqq 0$.

Define $G_{0}\left(W_{\mathscr{R}}\right)$ (respectively $G_{0}^{+}\left(W_{\mathscr{R}}\right)$ ) to be the set of points $(x, t)$ (respectively, $(x, t, \xi, \tau))$ on $\mathscr{R}$ or on rays generated from $\mathscr{R}$ by various reflections at $\partial \Omega$. Set

$$
\begin{gathered}
G\left(W_{\mathscr{R}}\right)=G_{0}\left(W_{\mathscr{R}}\right) \cap(\partial \Omega \times(0, \infty)), \\
G^{+}\left(W_{\mathscr{R}}\right)=\left\{(x, t, \xi, \tau):(x, t) \in G\left(W_{\mathscr{R}}\right)\right\} .
\end{gathered}
$$

Definition 5.1. $G\left(W_{\mathscr{R}}\right)$ (respectively, $G^{+}\left(W_{\mathscr{R}}\right)$ ) is simple if each point in $G\left(W_{\mathscr{R}}\right)$ (respectively, $G^{+}\left(W_{\mathscr{R}}\right)$ ) corresponds to a unique incoming ray.

We shall assume that one of the following conditions holds.

$\left(\mathrm{A}_{1}\right)$ For every $T>0$ the set of $W$ for which $1<|x|<2$ and $T^{-1}<|t|<T$, and such that the restriction $G\left(W_{\mathscr{R}}\right)$ to $0<t<T$ is not simple, has measure zero, or

$\left(\mathrm{A}_{2}\right)$ The same as $\left(\mathrm{A}_{1}\right)$ except that $G$ is replaced by $G^{+}$.

The assumptions $\left(A_{1}\right)$ or $\left(A_{2}\right)$ will permit us to replace geometric optics by ray optics in our discussion by the simple device of choosing only initial rays $\mathscr{R}$ for which $G_{0}\left(W_{\mathscr{R}}\right)$ or $G_{0}^{+}\left(W_{\mathscr{R}}\right)$ is simple, and then taking initial data with very small support for the geometric optics problem.

In the sequel, when we discuss necessary conditions for $L_{2}$ to be a continuable initial condition, we shall automatically assume (4.2) and $\left(\mathrm{A}_{1}\right)$; if we consider homogeneous initial data and inhomogeneous boundary data (so that all estimates will be integrated with respect to $t$ over some interval $0 \leqq t \leqq T$ ), we shall assume (4.3) and $\left(\mathrm{A}_{2}\right)$.

Remark. In studying the well-posed nature of the ray optics problem under the above assumptions, we can assume without loss of generality that the reflection coefficients $R_{j}$ are all non-negative. This is because we shall neglect interactions and are only interested in the strengths of the rays.

\section{The Prolongation of Rays}

Given the system of equations (2.1), where $A_{i}(x, t)$ are constant $N \times N$ matrices, there is associated with it a homogeneous polynomial of degree $N$, called the characteristic polynomial, defined by

$$
p(\xi, \tau)=\operatorname{det}\left[\tau I-\sum_{i=1}^{n} A_{i} \xi_{l}\right], \quad \xi=\left(\xi_{1}, \ldots, \xi_{n}\right) .
$$

Since (2.1) is assumed to be hyperbolic, the zero set of $p(\tau, \xi)$ is a real algebraic variety $V$, the characteristic variety. Furthermore, the strict hyperbolicity of (2.1) implies that for fixed $\xi \neq 0 \in R^{n}, p(\xi, \tau)$ has $N$ real and distinct zeros $\tau_{j}$. It follows 
that for real $\tau \neq 0$, the variety

$$
V_{\tau}=\left\{\xi \in R^{n}: p(\xi, \tau)=0\right\}
$$

consists of $[N / 2]$ nested "ovals" $\mathfrak{O}_{j}$. Each of the inner $[(N-1) / 2]$ of these ovals contains the origin in its interior (by the interior of $\mathcal{O}_{j}$, we mean the union of those components of $\mathcal{O}_{j}^{c}$ which do not intersect every straight line). If all the ovals are closed, then the inner oval is convex, and the other ovals are star-shaped with respect to every point on the inner oval.*

It is important to note that some of these ovals can contain the point at infinity; i.e., some of these ovals may not be "closed". For example, if $n=2$, consider the polynomial

$$
p\left(\xi_{1}, \xi_{2}, \tau\right)=\left(-\tau-\xi_{1}\right)\left(\tau^{2}+2 \tau \xi_{1}-\xi_{2}^{2}\right) \prod_{k=2}^{M}\left\{\left(-\tau-\xi_{1}\right)^{2}+k^{-2}\left(\xi_{1}^{2}+\xi_{2}^{2}\right)\right\} .
$$

For fixed $\xi=\left(\xi_{1}, \xi_{2}\right) \neq(0,0)$, the roots of $p=0$ are $\tau=-\xi_{1},-\xi_{1} \pm k^{-1}|\xi|$, $k=1,2, \ldots, M$, which clearly are distinct. To see that $p$ is the characteristic polynomial of a system (2.1), we define the $2 \times 2$ matrices

$$
A_{k}=\left[\begin{array}{cc}
\alpha_{k} & 0 \\
0 & \beta_{k}
\end{array}\right], \quad B_{k}=\left[\begin{array}{cc}
0 & -k^{-2} \\
1 & 0
\end{array}\right]
$$

where $\alpha_{k}+\beta_{k}=-2, \alpha_{k} \beta_{k}=1-k^{-2}$, and set

$$
A=\left[\begin{array}{llll}
1 & & & \\
& A_{1} & & 0 \\
& & \ddots & \\
0 & & A_{M}
\end{array}\right], \quad B=\left[\begin{array}{llll}
0 & & & \\
& B_{1} & & 0 \\
& & \ddots & \\
0 & & B_{M}
\end{array}\right] .
$$

Then it is easy to check that $p$ is the characteristic polynomial of the operator $\partial_{t}-A \partial_{x}-B \partial_{y}$

Next, we note that a variety $V$ defined by $p(\xi, \tau)=0$ cannot have any singular points in $\tau \neq 0$. For if $V$ were singular at a point $P=(\bar{\xi}, \bar{\tau}), \bar{\tau} \neq 0$, then $\nabla_{\xi} p(P)=0$, $p_{\tau}(P) \neq 0$ (strict hyperbolicity), and $V p(P)$ would point along the $\tau$ axis. But $\bar{\tau}=0$ by the homogeneity of $p$. This result also shows that $V$ has a well-defined normal at each point. The following lemma is well-known.

Lemma 6.1. The reduced equation $\sum_{i=1}^{n} A_{i} \partial_{x_{i}} u=0$ is elliptic if and only if $V_{\tau}$ consists of closed ovals for some (and therefore all) $\tau \neq 0$.

The geometry of $V$ is intimately connected with the construction and prolongation of ray signals, including their reflections on the various surfaces $x_{i}=0,1 \leqq i \leqq n$. It is well-known that the direction of any ray is computed from the system of ordinary differential equations defining the bicharacteristics $\star \star$

$$
\dot{t}=p_{\imath}, \quad \dot{x}_{j}=p_{\xi_{j}}, \quad j=1,2, \ldots, n ;
$$

$\star$ In $R^{n} \sim\{\infty\}$ some ovals may be disconnected; cf. $\S 8$.

$\star \star$ Since we are assuming that (2.1) has constant coefficients we need not consider the equations $\dot{\xi}_{j}=-P_{x_{j}}, \dot{\tau}=-P_{t}$. 

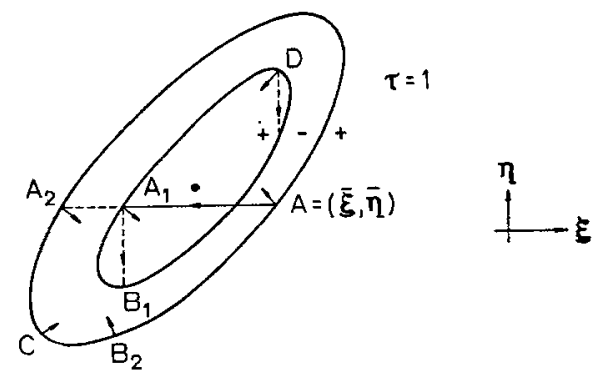

Fig. 1

naturally the rays travel along straight lines. Now as we have shown above $(\S 5)$, the direction of the ray is determined by the pair $(\xi, \tau)$ in the term $r \exp i(t \tau+x \cdot \xi)$. For fixed $\tau \neq 0,(6.2)$ defines either exterior normals for all the ovals (if $\tau<0$ ) or interior normals for all the ovals (if $\tau>0$ ). This is easily seen by using (6.2) to calculate sgn $d x_{i} / d t$; it depends on the sign change of $p$ as we cross the characteristic variety. Thus the directions of the rays are determined by the normals of $V_{\tau}$.

Now take any point $(x, t)$ in $\Omega \times\{t \geqq 0\}$ and any $(\bar{\xi}, \bar{\tau}) \in V, \bar{\tau} \neq 0$. We construct the bicharacteristic curve through $(x, t)$ associated with this given $(\bar{\xi}, \bar{\tau}){ }^{\star}$ We follow this ray until it hits the boundary, say at $x_{i}=0$. The reflected rays will be of the form $\sum c_{j} \exp i\left(t \tau+x \cdot \xi_{j}\right), \xi^{j}=\left(\xi_{1}^{j}, \ldots, \xi_{n}^{j}\right)$, and in order to satisfy the boundary condition

$$
P_{i}\left(r e^{i(t \bar{\tau}+x \cdot \bar{\xi})}+\sum c_{j} e^{i\left(t \bar{\tau}+x \cdot \xi^{j}\right)}\right)=0
$$

we must have $\xi_{k}^{j}=\bar{\xi}_{k}, k \neq i, j=1, \ldots, n$; here $\xi_{i}^{j}$ is free.

Thus in $\xi$-space (we have fixed $\tau=\bar{\tau} \neq 0$ ) we draw the line $\left\{\xi: \xi_{k}=\bar{\xi}_{k}, k \neq i\right\}$ and see where it meets the variety $V_{\bar{\tau}}$. This may be in several points, depending on the number of sheets of $V$. Suppose $\overline{\bar{\xi}}$ is such a point. Then there is a normal vector associated with $\bar{\xi}$ which, provided it points from $x$ into $\Omega$, determines the direction of a new reflected ray. We consider this process indefinitely; in general some prolongations of the given ray head to infinity while others are trapped.

In the case $n=m=2$ it is easy to picture the various reflections. Here we fix $\tau=1$ and use the notation $\left(\xi_{1}, \xi_{2}\right)=(\xi, \eta),\left(x_{1}, x_{2}\right)=(x, y)$. We suppose that $V_{1}$ consists of two closed nested ovals as shown in Figure 1. These ovals separate the $\xi-\eta$ plane into three regions, each of which determines a sign of $p$ (we have chosen one such "sign pattern" in our figure). The ray points along the inner normal at $A$, where $p_{\tau}<0, p_{\xi}>0$ and $p_{\eta}<0$; this implies that $d x / d t<0, d y / d t>0$. It follows that the ray points inward along the entire outer oval. Similarly, the normals point inward along the entire inner oval.

Now we consider a ray through a point $(x, y, t)$, and we construct the bicharacteristic curve associated with this point and $(\bar{\xi}, \bar{\eta}, 1)$. Suppose that our ray starts on the plane $y=0$; since the normal at $A$ points toward the plane $x=0, \star \star$

* If $(\xi, \tau) \notin V$, then the matrix $p(\xi, t)$ has no null space, and the Ansatz described in $\S 5$ simply fails; thus in what follows, we always take $(\xi, \tau) \in V$.

$\star \star$ Unless otherwise noted, we shall always assume our corner is $x_{i} \geqq 0, t \geqq 0$. If this is not the case, our discussion must be modified slightly. 


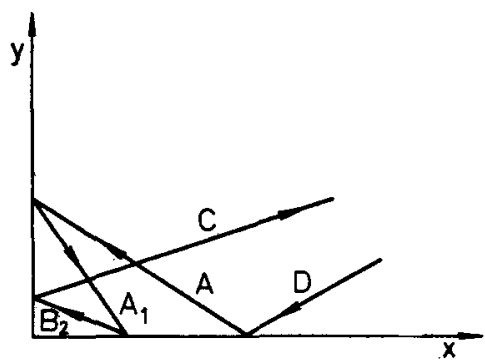

Fig. 2

this ray impinges on $x=0$ and we are free to choose a new $\xi$. The possibilities here are the two points $A_{1}=\left(\xi_{1}, \bar{\eta}\right)$ and $A_{2}=\left(\xi_{2}, \bar{\eta}\right)$. Each of these points determines a reflected ray which prolongs the original ray. Suppose we consider $A_{1}$, where the normal points toward the plane $y=0$; the prolongation hits this plane, and we are free to choose a new $\eta$. The only possibilities are at $B_{1}$ and $B_{2}$; suppose we choose $B_{2}$. Now the only possibility is to go to the point $C$. But since the normal at $C$ does not point towards the boundary, the ray escapes to infinity. We depict this particular ray's history in Figure 2, where we have assumed, for definiteness, that the ray started from infinity with $(\xi, \eta)=D$ and hit the boundary at $y=0$. The ray path is $D \rightarrow A \rightarrow A_{1} \rightarrow B_{2} \rightarrow C$; the points $A, A_{1}, B_{2}, C$ are "reflections" in the variety $V_{1}$.

The history of this ray is quite straightforward, for the reasons that $n=2$ and the geometry of $V_{1}$ is rather simple. Note that if $n=2$ the direction alone of a reflected ray determines which, if any, of the boundary surfaces are hit. In higher dimensions, two reflected rays with the same direction may hit different boundary surfaces, depending on the starting point. Finally, we shall show in $\S 7$ that even in the case $n=2$ the characteristic variety can be chosen so as to force much more complicated ray patterns.

\section{Trapped Rays}

By a trapped ray, we mean a ray which is forever reflected in one or both directions off the various boundary surfaces: it does not escape to infinity, in one or both directions. As a special class of trapped rays, we can consider periodic rays, that is, rays which start at a point $P \in V_{\tau}$, reflect off boundary surfaces, and then return to $P$, after a finite number of reflections. In this section we shall prove the existence of such rays, and study some of their properties.

As we have seen earlier, the prolongation of rays, including their various reflections in the boundary, are connected with the geometry of the associated characteristic variety $V$. In order to construct examples of trapped rays we must be able to construct a variety $V$ whose projection on $\tau=$ const. $\neq 0$ has certain properties. For the scalar case this is accomplished by a simple perturbation technique which we now describe. The more complicated case of first order systems will be treated later.

Consider the real algebraic variety $\tilde{V}$ in the $\xi, \eta$ plane, defined by the polynominal $p_{1}(\xi, \eta) p_{2}(\tau, \eta)=0$, where $p_{1}$ and $p_{2}$ are second degree polynomials 


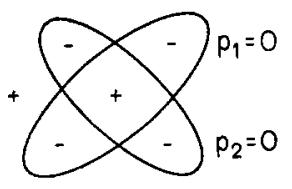

(a)

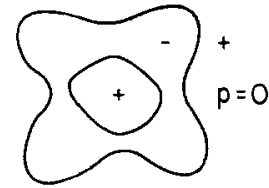

(b)

Fig. 3

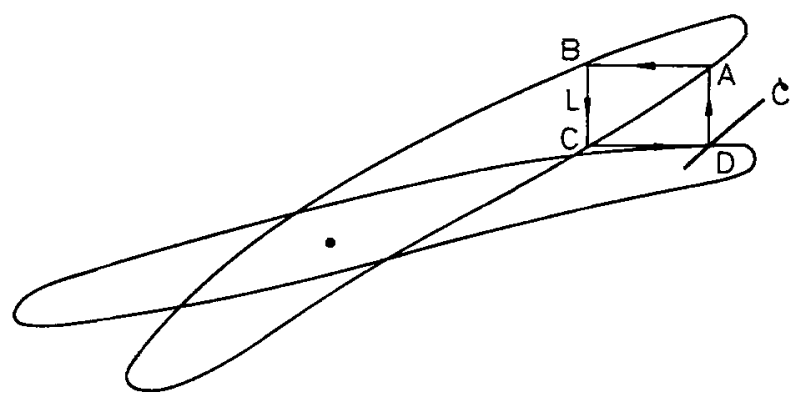

Fig. 4

whose associated varieties are ellipses. We assume that these ellipses intersect in four points, as in Figure $3 \mathrm{a}$ above. We have indicated in this figure the sign of the product in the various regions of the $\xi, \eta$ plane.

It is clear that $\tilde{V}$ cannot represent a strictly hyperbolic polynomial. However, by considering a slightly perturbed polynomial,

$$
p(\xi, \eta)=p_{1}(\xi, \eta) p_{2}(\xi, \eta)-\varepsilon,
$$

where $\varepsilon>0$, it is easy to see that the variety $V$ has the form shown in Figure $3 \mathrm{~b}$. Moreover, the variety $\tilde{V}$ is well approximated by $V$, and, for sufficiently small $\varepsilon>0, p$ is a strictly hyperbolic polynomial.

This technique of "pulling apart" ellipses is basic to our construction of trapped rays. To illustrate, consider two ellipses $p_{1}=0, p_{2}=0$ centered at the origin, and inclined so that $p=p_{1} p_{2}=0$ has the periodic trapped ray $L$ depicted in Figure 4. To construct such a ray, we choose points $A, B, C$ on $p_{1}=0$ such that $B$ is a right angle; this uniquely determines $D$. Then we construct $p_{2}$ such that $D$ lies on $p_{2}=0$, as depicted. We may carry out the same construction starting with points $\bar{A}$ near $A$ on $p_{1}=0$; the locus of points $\bar{D}$ forms a curve $\mathscr{C}$ containing $D$. Finally, we can arrange it that $\mathscr{C}$ meets $p_{2}=0$ transversally at $D$. If we now consider $q=p-\varepsilon$, for small $\varepsilon>0$, then the corresponding curve $\mathscr{C}_{\varepsilon}$ cuts the outer oval of $q=0$ transversally at a point near $D$. This implies that the polynomial $g=0$ admits a periodic ray.

The only shortcoming in this technique is that it is unclear how to associate this polynominal with a first-order system. To remedy this, we need the following lemma. 


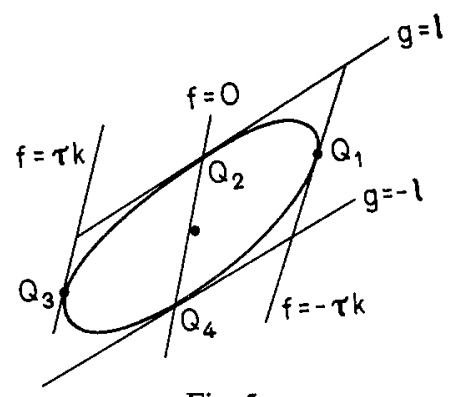

Fig. 5

Lemma 7.1. Given an ellipse $p(\xi, \eta)=0$, a real number $\tau \neq 0$, and finitely many points $P_{1}, \ldots, P_{r}$, we can find linear functions

$$
f=a_{1} \xi+b_{1} \eta+c_{1} \tau, \quad g=a_{2} \xi+b_{2} \eta+c_{2} \tau
$$

such that the given ellipse is in the form $f^{2} / k^{2}+g^{2} / l^{2}=\tau^{2}$ for some real $k, l, k l \neq 0$, and no $P_{i}$ lies on either $f=0$ or $g=0$.

Proof. We first find two diametrically opposed points $Q_{1}, Q_{3}$ on the given ellipse such that the tangents through $Q_{1}$ and $Q_{3}$ do not contain any of the points $P_{j}, 1 \leqq j \leqq r$. These tangent lines are described by the equations $f=\tau k$ and $f=-\tau k$. We construct the line $f=0$, passing through the center of the ellipse and meeting the ellipse at points $Q_{2}, Q_{4}$. Similarly, we construct tangent lines $g= \pm l$ at $Q_{2}, Q_{4}$ respectively. By changing $Q_{1}$ slightly, we can be sure that none of the lines $f= \pm \tau k, g= \pm k l$ meet any of the $P_{j}$ 's. Then it is easy to see that the given ellipse is of the desired form. (The construction is shown in Figure 5.)

We can now construct a first-order system whose characteristic polynomial has the desired properties; this follows from

Proposition 7.2. Let $p=p_{1} \ldots p_{t}$ where each $p_{i}=p_{i}(\xi, \eta)$ is a quadratic function whose corresponding real algebraic variety defines an ellipse containing the origin. Suppose that each of these ellipses is contained in a given set $K: \xi^{2}+\eta^{2} \leqq r^{2}$. Then for any $\delta>0$, there exists a strictly hyperbolic first order system of $2 t$ equations in $2 t$ unknowns whose characteristic polynomial $\tilde{p}$ satisfies $|p-\tilde{p}|<\delta$ on $K$.

Proof. By a small perturbation, we can place the ellipses $p_{i}=0$ in general position, that is, no two of the $p_{i}$ 's have a common factor and no point lies on more than two of the ellipses. We again call the resulting characteristic polynomial $p$.

We shall perturb $p$ by adding to it a function $G(\xi, \eta)$ which is so small that $V_{\tau}$ is perturbed slightly except near the intersection points of the ellipses; moreover, $G$ will have the proper sign to pull them apart in the desired way. This sign can be determined by the following algorithm: $V_{\tau}$ is, topologically, a finite disjoint union of simply connected arcs $\overline{P_{j} P_{k}}$ and double points $P_{j}$ (Figure 6a). Let $\sigma$ denote the sign of $p$ in the exterior $\mathscr{E}$ of $V_{\imath}$, and set $\operatorname{sgn}\left(G\left(P_{j}\right)\right)=-\sigma$ if $P_{j}$ touches $\mathscr{E}$. Next define $V_{\tau}^{\prime}=V_{\tau} \sim \cup\left(\overparen{P_{j} P_{k}}\right)$ (Figure 6b), where the union is 


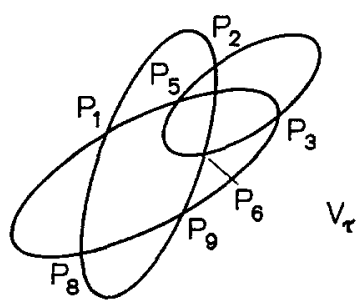

(a)

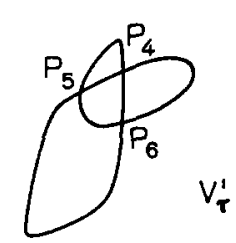

(b)

Fig. 6

taken over all $\operatorname{arcs}{\overparen{P_{j} P_{k}}}_{k}$ which border on $\mathscr{E}$. Now describe $V_{\tau}^{\prime}$ just as before as a union of points $P_{j}^{\prime}$ and $\operatorname{arcs} \widehat{P_{j}^{\prime} P_{k}^{\prime}}$, and set $\operatorname{sgn} G\left(P_{j}^{\prime}\right)=+\sigma$ if $P_{j}^{\prime}$ touches the exterior of $V_{\tau}^{\prime}$. This process may be continued until $\operatorname{sgn} G$ is determined at all the $P_{j}$ 's. We shall now construct $\tilde{p}$.

By Lemma 7.1 we can write, for fixed $\tau, \tau \neq 0$,

$$
p=\operatorname{det}\left[\begin{array}{lll}
A_{11} & & \\
& \ddots & \\
& & A_{t t}
\end{array}\right],
$$

where each of the $A_{j j}$ are $2 \times 2$ matrices of the form

$$
A_{j j}=\left[\begin{array}{cc}
\tau-f_{j} / k_{j} & g_{i} / l_{i} \\
g_{j} / l_{j} & \tau+f_{j} / k_{j}
\end{array}\right]
$$

and no entry vanishes at any of the intersection points $\left\{p_{r}=0\right\} \cap\left\{p_{s}=0\right\}$, $1 \leqq r, s \leqq t$.

We first consider the $(4 \times 4)$ submatrix defined by $A_{11}$ and $A_{22}$. We choose linear functions $a_{12}, b_{12}$ so that $\operatorname{sgn}\left(a_{12} b_{12}\right)$ is correct at each of the (at most) four intersection points of $p_{1}=0$ and $p_{2}=0$. The new perturbed submatrix we take to be

$$
\left[\begin{array}{cccc}
\tau-f_{1} / k_{1} & g_{1} / l_{1} & 0 & 0 \\
g_{1} / l_{1} & \tau+f_{1} / k_{1} & \varepsilon b_{12} & 0 \\
0 & \varepsilon a_{12} & \tau-f_{2} / k_{2} & g_{2} / l_{2} \\
0 & 0 & g_{2} / l_{2} & \tau+f_{2} / k_{2}
\end{array}\right]
$$

Observe that its determinant is $p_{1} p_{2}+\varepsilon^{2} a_{12} b_{12}\left[q_{12}\right]$, where $q_{12}$ is a homogeneous quadratic function which is bounded in $K$ and where $\operatorname{sgn}\left(a_{12} b_{12} q_{12}\right)$ "pulls apart" the varieties defined by $p_{1}=0$ and $p_{2}=0$. Here $\varepsilon \geqq 0$ is a small parameter; we take $\varepsilon=0$ if and only if $p_{1}=0$ and $p_{2}=0$ have no intersection points. Observe that we have only added terms in the upper right $\left(A_{12}\right)$ and lower left $\left(A_{21}\right)$ entries.

The technique which we have described is general. Thus, in order to "pull apart" the intersection points of $p_{i}=0$ and $p_{j}=0,1 \leqq i \leqq j \leqq t$, we consider the 
matrices $A_{i i}$ and $A_{j j}$ and the corresponding $2 \times 2$ matrices $A_{i j}$ and $A_{j i}$. We define

$$
A_{i j}=\left[\begin{array}{cc}
0 & 0 \\
\varepsilon b_{i j} & 0
\end{array}\right], \quad A_{j i}=\left[\begin{array}{cc}
0 & \varepsilon a_{i j} \\
0 & 0
\end{array}\right],
$$

where $\varepsilon \geqq 0$ and $\operatorname{sgn}\left(a_{i j} b_{i j}\right)$ is correct at each of the (at most) four intersection points of $p_{i}=0$ and $p_{j}=0$. Note that this does not destroy any other "pulling apart" process, since the contribution in the characteristic polynomial due to $A_{i j}$ and $A_{j i}$ is multiplied by a factor which is zero at the other intersection points. Let $\tilde{p}$ denote the determinant of this new matrix; then

$$
\tilde{p}=p+\varepsilon^{2} \sum_{1 \leqq i<j \leqq t}\left(a_{i j} b_{i j}\right) q_{i j},
$$

where $q_{i j}$ is a homogeneous polynominal of degree $2 t$. This completes the proof of the proposition.

Remark. A similar construction can be carried out if the degree of $p$ is odd, say $2 t+1$, provided that $p$ has a single linear factor $p_{0}$ whose real zeros do not meet those of the quadratic factors $p_{i}, 1 \leqq i \leqq t$. Thus (7.1) is replaced by

$$
p=\operatorname{det}\left[\begin{array}{llll}
A_{00} & & & \\
& A_{11} & \\
& & \ddots & \\
& & A_{t t}
\end{array}\right],
$$

where $A_{00}$ is a $1 \times 1$ matrix consisting of a single linear factor. The above construction is then repeated on the submatrix defined by $\operatorname{diag}\left(A_{11}, \ldots, A_{t}\right)$.

By means of Proposition 7.2 we can construct real plane algebraic varieties rather easily: we have only to take care that our constructions can be obtained by "pulling apart" ellipses.

\section{Some Qualitative Results on Trapped Rays}

In this section we shall construct some examples of trapped rays, periodic rays, and "limit cycles." We shall also discuss the stability of such trapped rays, and prove some interesting propositions concerning trapped rays. Finally we shall construct an example of a strictly hyperbolic first order system with "correct" boundary conditions, for which the geometric optics approximation, and consequently the full boundary value problem, is not well posed (Theorem 4.3).

Example 1. A periodic ray where the reduced equation is elliptic; $n=2, N=4$. This example has already been discussed in the previous section, where the characteristic polynomial did not come from a first-order system. However, in view of Proposition 7.2, we can assert the existence of such a periodic ray which comes from a first order system. Lemma 6.1 implies that the corresponding reduced equation is elliptic.

Observe that the inclination of the real algebraic variety plays a crucial role in the existence of trapped rays. For example, consider Figures $7 a, b, c$. In Figure $7 \mathrm{a}$, we have indicated the periodic ray together with those points $\Phi$ of 


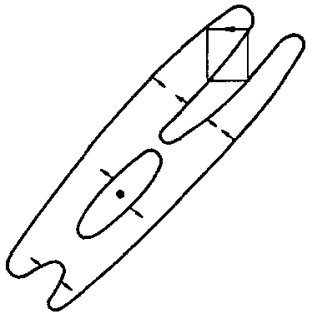

(a)

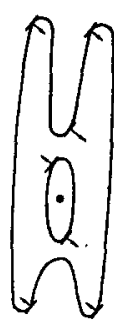

(b)

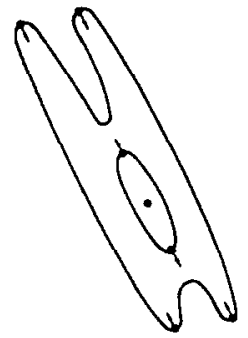

(c)

Fig. 7

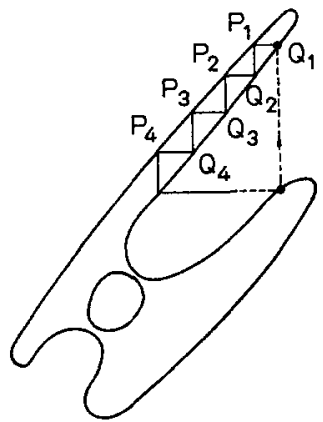

Fig. 8

the variety in which the inner* normals point into the 2 nd and 4th quadrants. As we rotate the entire figure in the counter-clockwise direction, $\Phi$ steadily decreases as indicated in Figures $7 \mathrm{~b}$ and $7 \mathrm{c}$. Clearly one wishes to maximize $\Phi$ in order to increase the likelihood of trapped rays. Observe that by a slight modification of our construction we can produce examples in which the periodic ray is reflected any number of times off different points at the boundary. For example, in Figure 8 since the normals at all of the $P_{i}$ 's as well as at all of the $Q_{i}$ 's are all different, the indicated periodic ray has the desired properties.

Propositon 8.1. There exist trapped rays, with $N=2$ and $N=3$, for which the reduced equation is not elliptic. If the characteristic variety $V_{1}$ includes a closed oval there cannot exist trapped rays for $N=2$ or $N=3$.

Proof. Consider a first order strictly hyperbolic 3-by-3 system whose corresponding variety is depicted in Figure 9. The trapped ray is indicated in the picture. Such a variety can easily be constructed; for example we can use the first two factors of $p$ defined in (6.1) and then rotate coordinates. Note that since the slopes of the normals at $Q_{i}$ decrease with increasing $i$, the trapped ray approaches the corner. By removing the linear factor, we see that this example works equally

$\star$ For $\tau>0$ (respectively, $\tau<0$ ), it is easy to show that the desired normals always point into the interior (respectively, exterior) of the outer oval. 


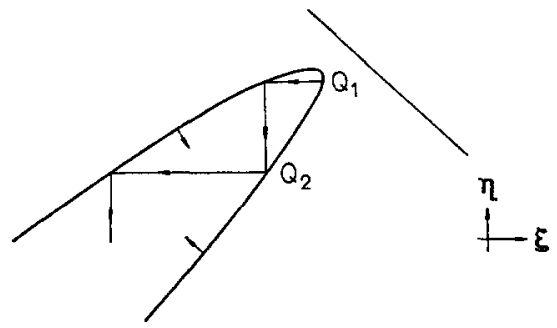

Fig. 9

well for $N=2$. We have considered detailed examples only for the case $n=2$; clearly such examples can be given for arbitrary $n$ and $m$ by rotating the given figure.

We now discuss conditions under which there is a bound on the total number of reflections along any ray path. Consider first the special case in which $V_{\tau}$ is an ellipse, $\tau \neq 0$.

Lemma 8.1. Let $n=m=N=2$, and suppose that the reduced equation is elliptic. Then there is a number $K$ such that no ray path has more than $K$ reflections.

Proof. By Lemma 6.1, the variety $V_{\tau}=\{(\xi, \tau): p(\xi, \tau)=0\}$ consists of a single closed oval which, since $N=2$, is an ellipse. Without loss of generality, suppose that $\tau>0$ so that the ray associated with $\xi \in V_{\tau}$ moves in the direction of the inner normal $n(\xi)$. Let a ray from $\Gamma_{2}$ hit $\Gamma_{1}$ and be reflected back to $\Gamma_{2}$. Then the associated points on $V_{\tau}$, namely $\xi^{1}$ and $\xi^{2}$, and the corresponding inner normals, $n_{1}$ and $n_{2}$, satisfy $\xi_{2}^{1}=\xi_{2}^{2}$ and $(-1)^{i+j} n_{j}^{i}<0, i, j,=1,2$. It follows easily that $\xi_{1}^{1}<\xi_{1}^{2}$. Similarly, if a ray from $\Gamma_{1}$ to $\Gamma_{2}$ is reflected back to $\Gamma_{1}$, then $\xi_{2}$ decreases. Thus $\xi_{1}$ and $\xi_{2}$ are monotonic on ray paths.

With $\xi, \eta \in R^{2}$, we shall write $\xi<\eta$ if $\xi_{1}<\eta_{1}, \xi_{2}<\eta_{2}$ and $\xi_{1}+\xi_{2}<\eta_{1}+\eta_{2}$. Let $\xi^{1} \in V_{\tau}$ have the maximum possible first coordinate $\xi_{1}^{1}$, and define a sequence $\xi^{1}, \ldots, \xi^{k}$ by the relations

$$
V_{\tau} \ni \xi^{j+1}<\xi^{j}, \quad \xi_{1}^{2 j}=\xi_{1}^{2 j-1}, \quad \xi_{2}^{2 j+1}=\xi_{2}^{2 j} ;
$$

the series ends at a point $\xi^{k}$ where the inner normal $n$ to $V_{\tau}$ satisfies $n \geqq(0,0)$. If the sequence $\eta^{j} \in V_{\tau}$ is associated with a ray, the series ends at a point $\xi^{k}$ where the inner normal $n$ to $V_{\tau}$ satisfies $n \geqq(0,0)$. Suppose the sequence $\eta^{j} \in V_{\tau}$ is associated with a ray originating at $\Gamma_{2}$; then $\eta^{j} \leqq \xi^{j}$ unless $\eta^{j} \geqq(0,0)$, in which case the ray escapes. Thus no ray which originates at $x_{2}=0$ can undergo more than $k$ reflections. Similarly, there is an upper bound $k^{\prime}$ on the number of reflections on a ray path starting at $\Gamma_{1}$, and hence no ray path contains more than $K=\max \left\{k, k^{\prime}\right\}$ reflections.

We now present a more general result. Let the variety $V_{\tau}, \tau \neq 0$, defined by the strictly hyperbolic polynomial $p$ consist of closed ovals, each of which is axially convex (by this we mean that any line parallel to a coordinate axis intersects each oval in at most two points). We introduce functions $f_{j}(\xi), j=1, \ldots, n$, defined as the distance from $\xi \in V_{\tau}$ to the nearest point $\xi+c e_{j} \in V_{\tau}$, where $e_{j}$ is a unit vector in the direction on the $j^{\text {th }}$ coordinate axis. If there is no such point 
we put $f_{j}=0$. Let $d$ be the diameter of $V_{\tau}$, and define

$$
h\left(V_{\tau}\right)=d^{-2} \inf _{\xi \in V_{\tau}} \min _{1 \leqq j \leqq n}\left\{f_{j}^{2}(\xi)+\left(\frac{\partial^{2}}{\partial \xi_{j}^{2}} p(\xi)\right)^{2}\right\} .
$$

Proposition 8.2. Let $n$ and $N$ be fixed. Then for each $\varepsilon>0$ there is a nonnegative integer $K=K(\varepsilon, n, N)<\infty$ such that no ray can undergo more than $K$ reflections if $h\left(V_{\tau}\right)>\varepsilon$. In particular, if $h\left(V_{\tau}\right)>0$, there are no trapped rays.

Corollary. If the ovals in $V_{\tau}, \tau \neq 0$, are each uniformly convex, then there is a $K<\infty$ such that no ray path contains more than $K$ reflections.

Proof of Proposition 8.2. We claim first that there is monotonicity on ray paths; in particular, if $\tau>0$ (respectively, $<0$ ), then on each reflection some $\xi_{j}$ decreases (respectively, increases). For, with $\tau>0$, say, let $\xi^{1}$ and $\xi^{2} \in V_{\tau}$ be associated with an incident ray on $\Gamma_{j}$ and with one of its reflections. Then $\xi_{i}^{1}=\xi_{i}^{2}, i \neq j$, and the inner normals $n^{1}$ and $n^{2}$ to $V_{\tau}$ at $\xi^{1}$ and $\xi^{2}$ respectively, satisfy $n_{j}^{1}<0, n_{j}^{2}>0$. Because each oval of $V_{\tau}$ is axially convex, this implies $\xi_{j}^{1}>\xi_{j}^{2}$. Similarly, if $\tau<0, \xi_{j}^{\mathbf{1}}<\xi_{j}^{\mathbf{2}}$.

The proof continues by induction on $n$. Without loss of generality, assume that $d=1$. The conclusion follows from the following two observations:

(i) Each cross-section $V_{\tau}\left(\xi_{0}, j\right)$ of $V_{\tau}$ of the form $\left\{\xi \in V_{\tau}: \xi_{j}=\left(\xi_{0}\right)_{j}\right\}$ is either a single point or has associated with it an $h$ such that $h\left(V_{\tau}\left(\xi_{0}, j\right)\right) \geqq h\left(V_{\tau}\right)$.

(ii) Given $N>1$, and $n, h\left(V_{\tau}\right)>\varepsilon$ and $d=\tau=1$, there exists $\delta>0$ such that to each $\xi \in V_{\tau}$ there is a $j=j(\xi)$ for which $f_{j}(\eta)>\delta$ for all $\eta \in V_{\tau}$ satisfying $0 \leqq\left|\eta_{i}-\xi_{i}\right| \leqq \delta$, $1 \leqq i \leqq n$. The uniformity in (ii) follows easily from the following three facts, namely

(a) for fixed $p, V_{\tau}$ is compact,

(b) with $p$ normalized as $\tau^{N}+\sum_{|\alpha|=1}^{N} a_{\alpha} \xi^{\alpha_{\tau}} \tau^{N-|\alpha|}$, the set $\left\{a_{\alpha}\right\}$ of coefficients of $p$ is constrained to lie in a compact set $S$, and

(c) for $\tau \neq 0$ and $\left\{a_{\alpha}\right\} \in S, V_{\tau}$ depends smoothly on $\left\{a_{\alpha}\right\}$.

Now consider a ray associated with the point $\xi \in V_{\tau}$ and let $j=j(\xi)$ be as described in (ii). By the inductive hypothesis and (i), there cannot be more than $K(\varepsilon, n-1, N)$ successive reflections of this ray across faces different from $x_{j}=0$. Therefore, by (ii), after $K(\varepsilon, n-1, N)+1$ reflections at least one coordinate $\xi_{k}$ has changed by an amount $\delta$. Because of the monotonicity associated with axial convexity we conclude that $K(\varepsilon, n, N) \leqq\left(1+\delta^{-1}\right)(K(\varepsilon, n-1, N)+1)$.

Remark (a) If $n=2$, and if we require axial convexity with respect to just one of the coordinates rather than both, we can still conclude that there is a maximum number of reflections on any ray path since the monotonicity in just one $\xi_{j}$ will suffice.

Remark (b). One would expect the associated boundary value problem to be simpler if, in addition to the non-existence of trapped rays, we require that rays which have already been reflected off the boundary do not "formally" stimulate glancing rays on further reflection. If this is to hold true, it must be made an explicit assumption. 


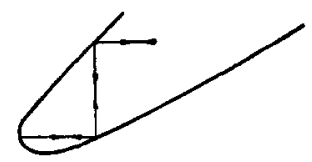

Fig. 10

Remark (c). If $V=V_{\tau}$ is an ellipsoid (the case $N=2$ ), the existence of a maximum number $K_{V}$ of reflections on a ray path has a simpler proof, which we sketch. First make the inductive hypothesis that each ellipsoid $V \subset R^{n-1}$ has a $K_{V}$. With $V \subset R^{n}$, then each non-trivial cross-section $V_{j}(s)=\left\{V \cap\left(\xi_{j}=s\right)\right\}$ has an associated $K_{j}$, and $K_{j}$ is independent of $s$, since $V_{j}(s)$ is similar to $V_{j}\left(s^{\prime}\right), s^{\prime} \neq s$. Thus after $\max K_{j}+1$ reflections, each $\xi_{j}$ has changed by an amount $\Delta \xi_{j} \neq 0$; moreover, $\max _{j}\left|\Delta \xi_{j}\right|$ is bounded away from zero. Using monotonicity as above, we obtain the required conclusion.

We remark that the strictly hyperbolic system as depicted in Figure 9 does not always admit a trapped ray; it depends on the particular corner.

With $\tau>0$, consider the parabolic variety depicted in Figure 10 . If $\Omega$ is the first (respectively third) quadrant, then rays are trapped forwards (respectively backwards). If $\Omega$ is the second or fourth quadrant, no ray is trapped.

We shall complete our discussion of trapped rays for $2 \times 2$ systems $(N=2)$ in two space variables $(n=2)$. We have seen that when the variety $V_{\tau}, \tau \neq 0$, is an ellipse (so that the reduced equation is elliptic by Lemma 6.1), then there is a number $K$ such that no ray can undergo more than $K$ reflections in the boundary; there are no trapped rays.

Suppose next that $V_{\tau}$ is a parabola. Then $V_{\tau}$ contains the origin in its interior, and the associated rays point into the interior. If trapped rays are to exist, then the axis of $V_{\tau}$ must lie in the first and third quadrants, and the parabola must look qualitatively like the one in Figure 9. By a change of scale, we can arrange for the axis of the parabola to make an angle of $5 \pi / 4$ with the positive $\xi$-axis. For simplicity, we take $\tau=1$.

Consider first the case where the axis of $V_{1}$ intersects the origin. Then $V$ and $V_{1}$ are defined respectively by the equations

$$
\begin{aligned}
& p(\xi, \eta, \tau)=-\tau^{2}+(\xi+\eta) \tau+(\xi-\eta)^{2}=0 \\
& p(\xi, \eta, 1)=(\xi-\eta)^{2}+(\xi+\eta)-1=0 .
\end{aligned}
$$

We start a ray from the point $x=1, y=0$, in a direction normal to $V_{1}$ at a point $(\xi, \eta)$, with $\xi>\eta, \xi<0$. The ray hits the $y$ axis at the point $(0, y)$, where

$$
y=-p_{\eta} / p_{\xi}=\frac{2(\xi-\eta)-1}{2(\xi-\eta)+1}=\frac{2 r-1}{2 r+1}
$$

and $r=\xi-\eta$. The reflected ray moves in a direction normal to $(\bar{\xi}, \eta)$, where $\bar{\xi}=\xi-(5-8 \eta)^{\frac{1}{2}}$, and hits the $x$-axis at the point $(\bar{x}, 0)$, where

$$
\bar{x}=\frac{(2 r-1)}{2 r+1} \cdot \frac{2(\bar{\xi}-\eta)+1}{2(\bar{\xi}-\eta)-1} .
$$



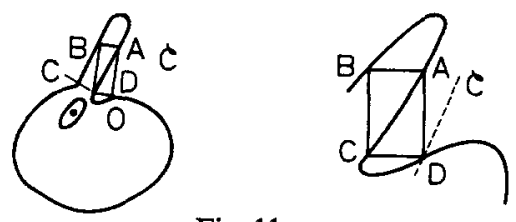

Fig. 11

One computes easily that $\bar{\xi}-\eta=-(r+1)$, and hence

$$
\bar{x}=\frac{2 r-1}{2 r+1} \cdot \frac{-2 r-1}{-2 r-3}=\frac{2 r-1}{2 r+3} .
$$

The reflected ray from the point $(\bar{x}, 0)$ moves in a direction normal to $(\bar{\xi}, \bar{\eta})$, where $\bar{\eta}=n-(5-8 \bar{\xi})^{\frac{1}{2}}=\eta-(3+2 r)$, so that $\bar{\xi}-\bar{\eta}=\xi-1-2 r-\eta+3+2 r=r+2$. At its $n^{\text {th }}$ return to the $x$-axis, then, the ray hits the point

$$
x_{n}=\frac{(2 r-1)}{(2 r+3)} \cdot \frac{(2 r+3)}{(2 r+3)} \cdots \frac{(2 r+4 n-5)}{(2 r+4 n-1)}=\frac{2 r-1}{2 r+4 n-1},
$$

which clearly tends to zero. However, the ray's approach to the corner is slow; the length of the $n^{\text {th }}$ circuit is of order $n^{-1}$, the ray speed is of order $\left(\xi^{2}+\eta^{2}\right)^{-\frac{1}{2}}$ which itself is of order $n^{-2}$ so that the elapsed time on the $n^{\text {th }}$ circuit is of order $n$. Thus the ray arrives at the point $\left(x_{n}, 0\right)$, where $x_{n}$ is of the order $r n^{-1}$, at approximately the time $c n^{2}$. It approaches the $n^{\text {th }}$ point with speed $O\left(n^{-3}\right)$.

If the axis of the parabola $V$ does not intersect the origin, the above results still hold; the only difference is that the propagation speeds are modified by a factor which is asymptotically negligible.

Finally, suppose $V$ is a hyperbola. In this case, the ray direction follows the inner normal on the branch containing the origin, and the outer normal on the other branch. It is easy to see that if there is no maximum number of reflections, then the branches of the hyperbola lie between asymptotes whose directions for one branch lie in first quadrant and for the other branch in the third quadrant. It is easily verified that a ray on one branch is trapped and comes arbitrarily close to the corner (but does not reach it in any finite time), while a ray associated with the other branch moves away from the corner and eventually escapes.

We next consider some other qualitative features of trapped rays. First, we exhibit a "limit cycle" which is a trapped periodic ray with the property that all trapped rays tend to it as the number of reflections increases.

Example 2. A limit cycle where the reduced equation is elliptic $(n=2, N=4)$.

Consider the system whose corresponding variety has the form shown in Figure 11. The point 0 is a relative maximum of the outer oval. The only region which can support a periodic orbit is the region on the "spike". The given depicted periodic orbit is constructed as before (see the discussion in \$7). The curve $\mathscr{C}$ is the analogous curve of "closing points"; that is, corresponding points $\bar{D}$ where we take $\bar{A}$ near $A$ on the spike. Since $\mathscr{C}$ cuts the outer oval transversally, we see that the ray $A B C D$ is the only periodic ray. 


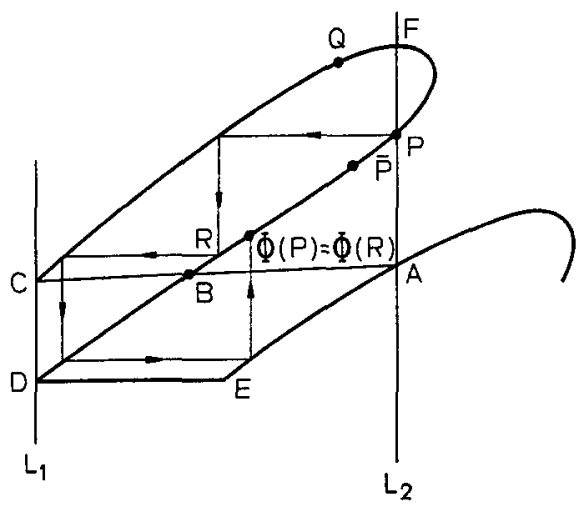

Fig. 12

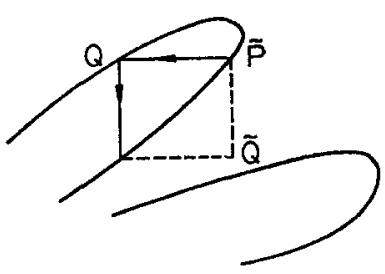

Fig. 13

Example 3. A trapped non-periodic ray where the reduced equation is elliptic; $(n=2, N=4)$.

Consider Figure 12, where $\overline{D E}>\overline{C B}$, and $L_{1}$ is parallel to $L_{2}$. The figure is a close up of a portion of a picture of two "pulled-apart" ellipses (cf. Figure 4). It is also drawn so that the ray starting at $P$ meets the upper branch $F C$ twice; then any ray starting at $P D$ meets $F C$ at most twice. Also, the lines $\eta=$ const. through any point on $P D$ cut the outer oval at points between $L_{1}$ and $L_{2}$. Finally, if we start at any point $\tilde{P}$ on $P R$ (the ray came from infinity before arriving at $P$ ) and follow a ray on its path, it must hit a point on $E A$ and then return to $P R$; that is, we get a (continuous) mapping $\phi$ from $P R$ into itself. We have arranged for the ray to hit $F C$ at most 2 times, $P D$ at most 4 times and $E A$ once. If the described ray is to be periodic, we must have $\phi^{k} \tilde{P}=\tilde{P}$ for some natural number $k$. However, we have two real numbers which we can adjust, namely $\varepsilon$, the "pulling apart" parameter (see $\S 1$ ), and $\delta$, a rotation angle of the second ellipse. We first rotate the second ellipse so that the "closing point" $\widetilde{Q}$ is at a positive distance from this second ellipse (see Figure 13). If we consider $\phi$ as a function of $\varepsilon$, $0<\varepsilon<\varepsilon_{0} \ll 1$, then the equation $\phi^{k}(\varepsilon) \tilde{P}=\tilde{P}$ can have at most a finite number of solutions $\varepsilon$. Thus there are at most a countable number of $\varepsilon$ in $\left(0, \varepsilon_{0}\right)$ which yield periodic rays, and we have only to avoid these.

\section{Trapped Rays and Poorly-Posed Problems}

In this section we prove our main result, namely, that the existence of trapped rays can destroy the well-posed nature of the mixed problem. However, we first prove 
that no such example is possible if $N=2$. This shows that the example of OsHER [24] is quite special.

From the theory of symmetric hyperbolic systems (cf. §3) we have

Proposition 9.1. Let $L$ be a symmetric hyperbolic system in a region $\Omega \times[0, T] \subset$ $R^{n} \times[0, T]$, possibly with edges and corners, and suppose that the boundary condition is strongly dissipative ${ }^{\star}$ on each face. Then the geometric optics approxtmation is well-posed.

Proof. Geometric optics approximate solutions are required to vanish near edges and corners, and hence estimates of the form (4.2) or (4.3) hold globally.

Now STRANG [39], [40] has shown that any $2 \times 2$ strictly hyperbolic system can always be written in symmetric hyperbolic form, and that if the problem is $L_{2}$-well-posed then the boundary conditions are dissipative. From KreIss' observation ([17], Main Theorem 2) that the set of boundary conditions for which condition $B$ holds is precisely the interior of the set for which the problem is wellposed in the sense of Hadamard, it follows ${ }^{\star \star}$ that if condition $B$ holds, then the boundary conditions must be strongly dissipative.

We turn to our main result: The construction of a $4 \times 4$ strictly hyperbolic system with $n=2$, whose reduced part is elliptic, which admits a periodic ray $C$; we shall construct boundary conditions satisfying condition $B$ (see $\S 3$ ) on each face for which the geometric optics solution blows up. Then Theorem 4.3 shows that the full mixed problem is poorly-posed.

The system we consider is defined in the region $\star \star \star t>0, x<0, y<0$, and is of the form

$$
L u=L\left(D_{t}, D_{k}, D_{y}\right) u=u_{t}+(A+\varepsilon C) u_{x}+(B+\varepsilon D) u_{y}=0,
$$

where the characteristic matrix has the form

We set

$$
L(\tau, \xi, \eta, \varepsilon)=\left[\begin{array}{cccc}
\tau+f & g & 0 & 0 \\
g & \tau+h & \varepsilon \sigma & 0 \\
0 & -\varepsilon \sigma & \tau+F & G \\
0 & 0 & G & \tau+H
\end{array}\right] \equiv L_{0}(\tau, \xi, \eta)+\varepsilon N(\xi, \eta)
$$

$$
L_{1}=\left[\begin{array}{cc}
\tau+f & g \\
g & \tau+h
\end{array}\right], \quad L_{2}=\left[\begin{array}{cc}
\tau+F & G \\
G & \tau+H
\end{array}\right],
$$

so that $L_{0}$ has the block form $L_{0}=\operatorname{diag}\left[L_{1}, L_{2}\right]$.

Let $V_{0}$ be the restriction of the characteristic variety of $L_{0}$ to $\tau=1$ :

$$
V_{0}=\left\{(\xi, \eta): \operatorname{det} L_{0}(1, \xi, \eta)=0\right\} .
$$

$\star$ Cf. the discussion at the end of $\$ 3$.

$\star \star$ For $2 \times 2$ symmetric hyperbolic systems, it is easy to show that the boundary of the set of dissipative boundary conditions is also the boundary of the set for which the problem is wellposed.

$\star \star \star$ The argument would be the same if we took the corner $t>0, x>0, y>0$ and then considered a different periodic trapped ray, namely the "mirror image" $C$ ' of $C$ which we can construct in the region $\xi<0, n<0$. 


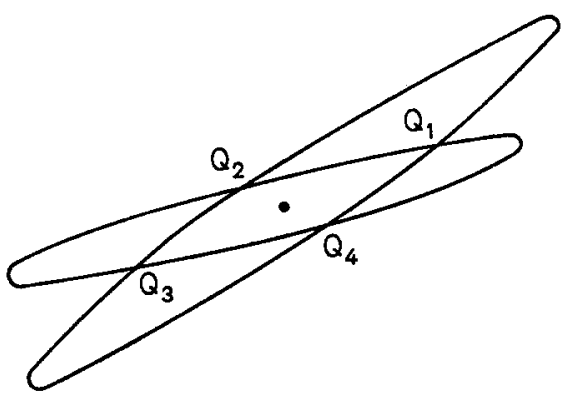

Fig. 14

Then $V_{0}$ consists of two intersecting ellipses $\mathscr{E}_{1}$ and $\mathscr{E}_{2}$ centered at the origin, both of whose major axes have positive slope, constructed as in $\S 7 . \mathscr{E}_{1}$ and $\mathscr{E}_{2}$ intersect in four points labeled $Q_{1}, Q_{2}, Q_{3}$, and $Q_{4}$ (see Figure 14). We shall study the behavior near $Q_{1}$ and $Q_{2}$; the other points are treated similarly. Note that the inner normals to $\mathscr{E}_{1}$ and $\mathscr{E}_{2}$ have a positive scalar product at $Q_{2}$ and a negative scalar product at $Q_{1}$.

The functions $f, g, h, F, G$, and $H$ are linear homogeneous functions of $(\xi, \eta, \tau)$; namely, $f=f_{1} \xi+f_{2} \eta+f_{3} \tau, g=g_{1} \xi+g_{2} \eta+g_{3} \tau$, etc., so that at $\tau=1$, $f=f_{1} \xi+f_{2} y+f_{3}$, etc. Furthermore, $\sigma$ is of the form $\sigma=\sigma_{1} \xi+\sigma_{2} \eta$.

These functions are chosen to satisfy the four conditions

i) $g\left(Q_{2}\right)=g\left(Q_{1}\right)=0$,

ii) $\sigma\left(Q_{i}\right) \neq 0, i=1,2,3,4$,

iii) $h\left(Q_{2}\right)=F\left(Q_{2}\right)=-1$,

iv) $f\left(Q_{t}\right) H\left(Q_{i}\right) \neq 0, \mathrm{i}=1,2,3,4$.

To achieve (i), we first construct a system of the form (9.2) with $f=-h, F=-H$ and $\sigma=0$, where $f, g, F$ and $H$ are functions of $\xi$ and $\eta$ only and are determined by the principal axes of the ellipses $\mathscr{E}_{1}$ and $\mathscr{E}_{2}$ (see $\S 7$ ). The resulting form is diagonalized at $Q_{2}$ by a similarity transformation. A reordering of the variables then yields (iii) since $L_{1}$ and $L_{2}$ are both singular on $\mathscr{E}_{1} \cap \mathscr{E}_{2}$. The remaining conditions are easily attained.

From the discussion in $\S 7$, the system (9.1) is strictly hyperbolic for small $\varepsilon>0$, and if the ellipses $\mathscr{E}_{i}, i=1,2$, are properly chosen, it admits a periodic ray, depicted in $\xi-\eta$ space (at $\tau=1$ ) by the corners $P_{1}^{\varepsilon}, P_{2}^{\varepsilon}, P_{3}^{\varepsilon}$, and $P_{4}^{\varepsilon}$, of a rectangle $R_{\varepsilon}$ (see Figure 15). Just one of these points lies near $\mathscr{E}_{1}$, say, and the others lie near $\mathscr{E}_{2}$.

Remark 9.1. Observe that we can easily arrange the geometry so that the periodic ray $P_{1}^{\varepsilon}-P_{2}^{\varepsilon}-P_{3}^{\varepsilon}-P_{4}^{\varepsilon}$ is a complete circuit; that is, if we follow a reflected ray that leaves the circuit, it can never return; the potential re-entry points $R, S, T$ and $U$ in Figure 15, are inaccessible to a ray starting on the circuit. To see this for $R, S$ and $T$, consider the region $\mathscr{R}$ to the left of $L K$ and above $K J$ where $L K$ (respectively $K J$ ) is a vertical (respectively horizontal) tangent to $V_{1}$, as drawn in Figure 15 . It is easy to see that any ray originating in $\mathscr{R} \cap V_{1}$ can never 


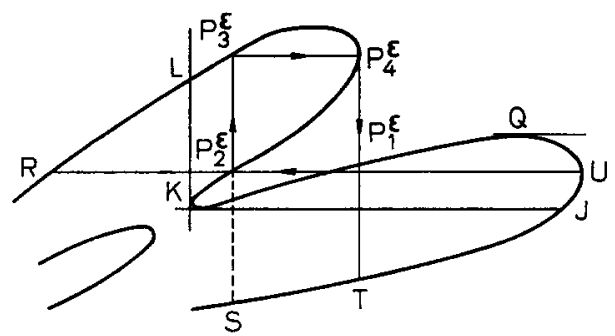

Fig. 15

leave this set since the reflected direction points outside of $\Omega$. For the point $U$ we can arrange that $U$ lies to the right of $Q$ where the tangent to $V_{1}$ at $Q$ is horizontal, and no point of $V_{1}$ lies above $Q$. Thus if the slope of $V_{1}$ at $U$ is negative, then the reflected ray goes to infinity without hitting $\Gamma$. If the slope of $V_{1}$ at $U$ is positive, then the ray at $U$ can only reflect to a point on $V_{1}$ between $Q$ and $U$, where the slope is negative, so we are back in the first case.

The boundary condition which we will apply at $x=0$ is of the form $P u=0$, where

$$
P=\left[\begin{array}{cccc}
1 & 0 & 0 & -k \\
0 & 0 & 0 & 0 \\
0 & 0 & 1 & 0 \\
0 & 0 & 0 & 0
\end{array}\right]
$$

with $k$ a large positive number. We now present

Lemma 9.1. Let $k$ be a fixed positive number. For sufficiently small $\varepsilon>0$, the boundary condition $P u=0$ on $x=0$ satisfies condition $B$.

Proof. We wish to show that the null space of $P$ and the space spanned by the "outgoing" vectors are bounded away from each other (in direction), uniformly for real $\eta$, and $\operatorname{Im} \tau \geqq 0$.

We claim first that this is true when $\varepsilon=0$. To see this, note that the timereduced systems obtained from $L_{1}$ and $L_{2}$ have eigenvalues \pm 1 , so that $f_{2}=H_{2}=-1$ and $h_{2}=F_{2}=-1$. It follows from the theory of symmetric positive systems ( $(3)$ that the homogeneous boundary conditions $u_{1}=\mathscr{F}, u_{3}=\mathscr{G}$ are well-posed for $L_{1}$ and $L_{2}$ respectively. Since $L_{1}$ and $L_{2}$ are uncoupled for $\varepsilon=0$, it follows that condition $B$ holds for this "unperturbed" system.

We must show that, for small $\varepsilon>0$, the mixed problem with boundary condition $P u=0$ is also well-posed at $x=0$.

First observe that the equation

$$
\operatorname{det} L_{1}(\xi, \eta, \tau)=\operatorname{det} L_{2}(\xi, \eta, \tau)=0
$$

is of the form

$$
a \xi^{2}+2 b \eta \xi+c \eta^{2}-\tau^{2}=A \xi^{2}+2 B \eta \xi+C \eta^{2}-\tau^{2}=0 .
$$


Since (9.4) has exactly four real solutions for $\tau$ real, $\tau \neq 0$, we conclude easily that solutions of (9.4) are of the form $\eta=(\alpha \pm \beta) \xi, \xi= \pm \gamma \tau$, with $\alpha, \beta, \gamma$ real, $\gamma \neq 0$, and where not both $\alpha$ and $\beta$ are zero. Consequently, for $\xi^{2}+(\operatorname{Re} \tau)^{2}+(\operatorname{Im} \tau)^{2}=1$, $\xi$ real, the characteristic roots $\eta$ of the equation

$$
\operatorname{det}\left[\check{\zeta}-B^{-1}(\tau+\eta A)\right] \equiv \operatorname{det}[\xi-M(\tau, \eta)]=0
$$

are uniformly bounded away from each other except in neighborhoods of real multiples of the points $(\xi, \tau)=( \pm \gamma, 1)$. Thus for complex $\tau$ and real $\eta$, with $|\tau|^{2}+\eta^{2} \neq 0$, the term $\varepsilon N$ perturbs the eigenspaces of $M(\tau, \eta)$ continuously in the complement $C_{N}$ of any conic neighborhood $N$ of $\bigcup_{i}\left(\xi\left(Q_{i}\right), 1\right)$; in any given $C_{N}$, then, condition $B$ is preserved provided $\varepsilon>0$ is sufficiently small.

Since the eigenvectors of $\xi-M(\tau, \eta)$ are independent of $\xi$, it suffices to consider small neighborhoods of the points $Q_{i}$, and $\tau$ near 1 . Observe that at the points $Q_{2}$ and $Q_{4}$, the rays (and eigenvectors) associated with $L_{1}$ and $L_{2}$ are both incoming or both outgoing, and in each case the eigenvectors of the opposite family are distinct from each other and span a complementary space. Thus the addition of a term of size ${ }^{\star} o(1)$ causes a small perturbation in the space spanned by the outgoing vectors.

It remains only to consider neighborhoods of $Q_{1}$ and $Q_{3}$. The argument is the same in each case and we consider only $Q_{1}$. For $(\xi, \eta, \tau)$ near $\left(Q_{1}, 1\right)$, and $0<|\varepsilon|<1$, we have

$$
L(\tau, \xi, \eta, \varepsilon)=\left[\begin{array}{cccc}
\tau+f\left(Q_{1}\right)+a & b & 0 & 0 \\
b & c & d & 0 \\
0 & -d & \alpha & \beta \\
0 & 0 & \beta & \tau+H\left(Q_{1}\right)+\gamma
\end{array}\right]
$$

where $|a|,|b|,|c|,|d|,|\alpha|,|\beta|$ and $|\gamma|$ are all $o(1)$ and $d \neq 0$. The eigenvectors of (9.6) have the form

$$
\begin{aligned}
& v_{1}=(1,0,0,0)+o(1) \\
& v_{2}=(0,0,0,1)+o(1) \\
& w_{i}=\left(0, p_{i}, q_{i}, 0\right)+(o(1), 0,0, o(1)), \quad i=1,2 .
\end{aligned}
$$

Furthermore, since the eigenvalues of a matrix depend continuously on its coefficients, the eigenvalues $\lambda_{i}$ corresponding to $\left(0, p_{i}, q_{i}, 0\right)$ are $o(1)$ in modulus. Since $v_{1}$ is outgoing and $v_{2}$ incoming, exactly one of the vectors $w_{i}$ is outgoing; call this vector $w$ and write it as $(0, p, q, 0)+o(1)$.

The null space of $P$ is spanned by the vectors $z_{1}=\left(1,0,0, k^{-1}\right)$, and $z_{2}=(0,1,0,0)$. To prove that the outgoing eigenspace and the nullspace of $P$ are bounded away from each other in direction, it suffices to show that for small $|\varepsilon|$ the determinant of the matrix whose columns are the vectors $v_{1}, w, z_{1}$ and $z_{2}$ is bounded away from zero for $(\tau, \xi, \eta)$ near $\left(1, Q_{1}\right)$. In view of $(9.7)$, this

\footnotetext{
$\star$ In this section $o(1)$ or $O(1)$ will always refer to $\varepsilon \rightarrow 0$.
} 
will be true if

$$
\operatorname{det}\left[\begin{array}{cccc}
1 & 1 & 0 & 0 \\
0 & 0 & 1 & p \\
0 & 0 & 0 & q \\
0 & k^{-1} & 0 & 0
\end{array}\right]
$$

is non-zero, so that we must show $q \neq 0$.

Suppose, to the contrary, that $q=0$. Then, since $(L-\lambda) w=0$, with $\lambda=o(1)$, we get an equation of the form

$$
\left[\begin{array}{cccc}
A & b & 0 & 0 \\
b & c & d & 0 \\
0 & -d & e & f \\
0 & 0 & f & B
\end{array}\right]\left(\begin{array}{c}
w_{1} \\
p \\
0 \\
w_{4}
\end{array}\right)=\left(\begin{array}{l}
0 \\
0 \\
0 \\
0
\end{array}\right)
$$

where, except for $p$, the lower case letters are $o(1), d \neq 0$, and $A$ and $B$, together with their inverses, are $O(1)$. From this we get successively from the fourth, third, and first equations of (9.8), that $w_{4}=0, p=0$, and $w_{1}=0$. Thus from the equation $L(\tau, \xi, \eta, \varepsilon) w=0$ with $(\tau, \xi, \eta)$ near $\left(1, Q_{1}\right), \varepsilon \neq 0$ and $w_{3}=q=0$, we conclude that $w \equiv 0$. This is a contradiction, and the lemma is proved.

We now show that the geometric optics solution corresponding to our periodic ray blows up. To this end, it is necessary to show that at $x=0$, the incoming signal corresponding to the point $P_{1}^{\varepsilon}$ is not annihilated by the matrix $P$. Thus, we must exclude the possibility that the incoming null vector for $L_{2}$ at $P_{1}^{z}$ is approximately $(0,1)$. Since $(0,1)$ is also a null vector at $Q_{1}$, we thus would have

$$
\left(1-\xi\left(Q_{1}\right) A-\eta\left(Q_{1}\right) B\right)\left[\begin{array}{l}
0 \\
1
\end{array}\right]=\left(1-\xi\left(P_{1}^{\varepsilon}\right) A-\eta\left(P_{1}^{\varepsilon}\right) B\right)\left[\begin{array}{l}
0 \\
1
\end{array}\right]=o(1)
$$

This implies that

$$
\operatorname{det}\left[\left(\xi\left(Q_{1}\right)-\xi\left(P_{1}^{\varepsilon}\right)\right) A+\left(\eta\left(Q_{1}\right)-\eta\left(P_{1}^{\varepsilon}\right)\right) B\right]=o(1),
$$

which either contradicts the ellipticity of the reduced operator $A \partial_{x}+B \partial_{y}$ or yields the contradiction $\left|Q_{1}-P_{1}^{z}\right|=o(1)$.

Similarly, the outgoing eigenvector at $P_{1}^{\varepsilon}$ is bounded away (in direction) from $(0,1,0,0)$, and has a first component bounded away from zero.

To trace a signal around the circuit $\left(\tau=1,(\xi, \eta)\right.$ going from $P_{1}^{\varepsilon}$ to $P_{2}^{\varepsilon}, P_{3}^{\varepsilon}, P_{4}^{\varepsilon}$ and back to $P_{1}^{\varepsilon}$ ), we need to impose a boundary condition at $y=0$. Using the fact that directions normal to $\mathscr{E}_{i}(i=1$ or 2$)$ at $Q_{1}$ or at $P_{i}^{\varepsilon}(i=1,2,3,4)$ which are incoming (respectively outgoing) at $x=0$, are outgoing (respectively incoming) at $y=0$, we see that an appropriate boundary condition at $y=0$ is $P u=0$, where

$$
P=\left[\begin{array}{cccc}
0 & 0 & 0 & 0 \\
0 & 1 & 0 & 0 \\
0 & 0 & 0 & 0 \\
0 & -k & 0 & 1
\end{array}\right]
$$

By arguments similar to those above, we see that (9.8) satisfies condition $B$ if $\varepsilon$ is sufficiently small. 
Finally, we give a signal which is initially incoming at $x=0$ and associated with $(\tau, \xi, \eta)=\left(1, P_{1}^{\ell}\right)$. (Note that the associated eigenvector $w$ is close to the space given by $w_{1}=w_{2}=0$.) If we trace such a signal around our circuit, then on each reflection its strength is multiplied by a number $s$ (depending on $P_{i}^{\ell}$ ) such that $\left|s^{-1}\right|=O\left(k^{-1}\right)$. Clearly, then, the signal has increased in strength after a complete circuit.

To complete the proof, we observe that the only way that blowup might be prevented is for some reflected ray to leave the circuit and then re-enter it; that is, in $\xi-\eta$ space, there might be points $R_{k}(1 \leqq k \leqq m)$ on $V_{1}$ such that there exists a ray $P_{i}-R_{1}-R_{2}-\cdots-R_{m}-P_{j}, 1 \leqq i, j \leqq 4$. But this too cannot happen in view of Remark 9.1.

A slight modification of our argument shows that (4.3) cannot hold either; namely, we have only to start our signal by means of an impulse given either by an inhomogeneous boundary condition or an inhomogeneous right-hand side (cf. Remark 2.1). We leave the details to the reader.

\section{Trapped Rays and Lacunas}

In this section we shall only be concerned with the case $n=2$, two space variables. This allows us to avoid the rather sophisticated topological techniques needed in the higher dimensional case ([41], [42]). Our main reference is [6], where the definition and sufficient condition for the existence of lacunas is given. Note that this condition applies to our case of first order systems ([6], p. 825).

Consider the real algebraic variety $V$ defined by $p(\tau, \xi, \eta)=0$, where $\xi, \eta \in R^{1}$ and $\tau \neq 0$ is fixed. We know that $V$ defines a finite number of nested ovals (some of which contain the point at infinity). The (geometric) condition for the existence of lacunas given in [6] is the following: let $\left(t_{0}, x_{0}, y_{0}\right)$ be a point in $R^{3}$; if the line $x_{0} \xi+y_{0} \eta=t_{0}$ meets $V$ in the maximum number of points, none of which is on the inner oval, then $\left(t_{0}, x_{0}, y_{0}\right)$ lies on a lacuna.

Before proving our main theorem, we shall give examples of some of the possibilities. Consider $(N=6)$ the varieties $V$ depicted in Figures 16a, b. In these figures, we can clearly arrange things so that we have a periodic trapped ray (cf. §7). However, Figure (16b) does not satisfy the above condition. Thus, it is not necessarily true that the existence of trapped rays implies the existence of lacunas (the converse is clearly false); however we do have the following theorem:
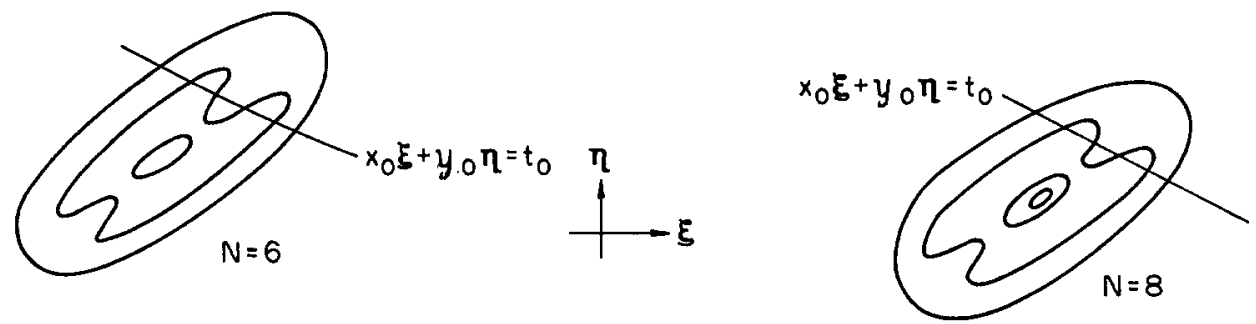

(a)

Fig. 16

(b) 
Theorem. For $N=4$, if there is a periodic trapped ray, then there exist lacunas along the coordinate axes.

Proof. It is easy to see that if there exists a periodic trapped ray, then there must exist points $P, Q, R$ on the variety with the property that $P$ is directly to the left of $Q, R$ is directly below $Q$, and the interior normals to $P$ and $R$ have negative second component, while the interior normal at $Q$ has a negative first component (otherwise the ray path is monotone (cf. $\S 8)$ ).

If $P$ and $Q$ do not lie on the same branch of $V$, then one is on the outer oval, and the other is on the inner; say that $Q$ lies inside the branch to which $P$ belongs. But then the line from $P$ to $Q$ meets $V$ in at least six points and this is impossible. Thus $P$ and $Q$ (and likewise $Q$ and $R$ ) lie on the same branch of $V$. It is then clear that this branch contains a point between $P$ and $Q$ with a vertical normal. Hence there is a lacuna along the $x$-axis; similarly there is a lacuna along the $y$-axis.

\section{Appendix-The Variable Coefficient Case}

We shall sketch the extension of the geometric optics solution of the mixed problem to the variable coefficient case. Our basic result is stated in two parts as

Theorem 11.1. (i) Theorem 4.3 is valid in the variable coefficient case. (ii) The geometric optics problem is well-posed if and only if it is well-posed in a neighborhood of each boundary point $(\bar{x}, \bar{t})$ for the constant coefficient problem with coefficients frozen at that point.

We shall prove (i) by showing that the geometric optics solution is asymptotic to the true solution. The claim (ii) follows from the continuous dependence of the geometric optics solution on the coefficients of the equation. We also use the fact that given an incoming ray, we can, by modifying the data ${ }^{\star}$, reduce the problem to one in so small a neighborhood of $(\bar{x}, \bar{t})$, (e.g. $\bar{x}$ at a corner) that the geometric optics solution approximates the constant coefficient geometric optics solution. Also, by looking in a very small neighborhood in $x-t$ space of a boundary point, we can ensure that real rays associated with the modified initial conditions develop no caustics within a given number of reflections. Note that the neighborhood may be very small if we exclude rays that are close to glancing. (Among other considerations, we want to exclude rays reflected twice in succession from the same face $\Gamma_{j} \cap U$. .)

In the following, we shall justify the validity of the geometric optics solution after a single reflection, in a domain possibly having corners and edges; the result extends immediately to multiple reflections.

We shall keep the notation of $\S 3,4$; in particular, we shall assume that reflection occurs along $\Gamma_{1}$ and that the boundary condition there is $P_{1} u=0$.

Suppose that an incoming signal is given as

$$
u_{i n}=e^{i \lambda \phi} a(x, t) r_{1}
$$

\footnotetext{
$\star$ e.g., choosing $\varepsilon$ small in (3.2).
} 
where $\phi$ and a satisfy (2.3) and (2.5), and $r_{1}$ is the associated null vector of $\mathscr{M}(\phi)$. We require that $\phi$ be real, that $a(x, 0)$ have small support, and that the associated bicharacteristics determined by (2.7) should hit $\partial \Omega$ at positive $t$.

At $x_{1}=0$, the incoming signal is

$$
u_{i n}\left(0, x^{\prime}, t\right)=e^{i \lambda \phi\left(0, x^{\prime}, t\right)} a\left(0, x^{\prime}, t\right) r_{1}\left(0, x^{\prime}, t\right)
$$

By applying a diffeomorphism in the $\left(x^{\prime}, t\right)$ variables, which is the identity outside of a neighborhood $\mathscr{H}$ of the support of $a\left(0, x^{\prime}, t\right)$, we ensure that $\phi\left(0, x^{\prime}, t\right)$ takes the form

$$
\phi\left(0, x^{\prime}, t\right)=x^{\prime} \cdot \bar{\eta}+t \tau
$$

in $\mathscr{H}$; outside of $\mathscr{H}$ we use (11.3) to redefine $\phi$.

The approximate outgoing solution must approximately satisfy the boundary condition $P u=0$. This induces the condition

$$
u_{\text {out }}\left(0, x^{\prime}, t\right)=e^{i \lambda \phi\left(0, x^{\prime}, t\right)} a\left(0, x^{\prime}, t\right)\left[\sum_{j>1} c_{j}\left(x^{\prime}, t\right) r_{j}\left(0, x^{\prime}, t\right)\right]
$$

where $r_{j}$ is a normalized right eigenvector or generalized eigenvector of $\mathscr{M}(\phi)$ with eigenvalue $\tilde{\xi}_{j}$. The reflection coefficients $c_{j}\left(x^{\prime}, t\right)$ are determined by the conditions $P\left(r_{1}+\sum_{j>1} c_{j} r_{j}\right)=o(1)$, and $c_{j}=0$ if $\operatorname{Im} \tilde{\xi}_{j}>0$ or if $\tilde{\xi}_{j}$ is real and the associated bicharacteristic is incoming.

If $\tilde{\xi}_{j}$ is real, then there is a real bicharacteristic associated with the dual vector $\left(\phi_{t}, \phi_{x^{\prime}}, \tilde{\xi}_{j}\right)$, and the outgoing geometric optics signal is found in a manner analogous to that of $\$ 2$.

In the sequel, we shall denote the variables dual to $\left(x_{1}, x^{\prime}\right)$ by $(\xi, \eta)$.

Suppose that $\tilde{\xi}_{j}$ is complex and that we are looking at a ray through the point $\left(0, x_{0}^{\prime}, t_{0}\right)$, with $\left(x_{0}^{\prime}\right)_{j}>0, j \neq 1$, and $t_{0}>0$. From $L u=0$, we get

$$
u_{x}=\mathscr{M} u, \quad \text { where } \mathscr{M}=A_{1}^{-1}\left(\frac{\partial}{\partial t}-A^{\prime} \cdot \frac{\partial}{\partial x^{\prime}}\right)
$$

Denote the symbol of $\mathscr{M}$ by $M=i A^{-1}\left(\tau-A^{\prime} \cdot \eta\right)$, and suppose that $\tilde{\xi}_{j}$ is an eigenvalue of $M$ with multiplicity $k_{0}$. We consider $\mathscr{M}$ as a pseudo-differential operator in $\left(x^{\prime}, t\right)$, parametrized by $x_{1}$.

To complete our treatment of the variable coefficient case, and in particular to justify both Theorem 4.3 and the geometric optics approximation for the mixed problem in a half-space, it will suffice to prove the following lemma.

Lemma 11.2. If $a\left(0, x^{\prime}, t\right) \in C_{0}^{\infty}\left(\Gamma_{1}\right)$ and has sufficiently small support containing $\left(0, x_{0}^{\prime}, t_{0}\right)$, then there exists a sequence $\left\{u_{\lambda}\right\}, \lambda \rightarrow \infty$, such that

(a) $\left\|u_{\lambda}\right\|+\left\|L u_{\lambda}\right\|=o(1) \quad$ as $\lambda \rightarrow \infty$,

(b) $\left\|P_{1}\left\{u_{\lambda}\left(0, x^{\prime}, t\right)-e^{i \lambda \phi\left(0, x^{\prime}, t\right)} a\left(0, x^{\prime}, t\right) \sum^{\prime} c_{j} r_{j}\right\}\right\|_{r_{1}}=o(1)$, 
as $\lambda \rightarrow \infty$, where the sum $\sum^{\prime}$ is taken over those $j$ for which $\xi_{j}\left(0, x_{0}^{\prime}, t_{0}, \bar{\eta}, \bar{\tau}\right) \neq \tilde{\xi}_{j}$, and

(c) $u_{\lambda}(x, t)=0$ if $t \leqq 0$ or $x \in U\left\{\Gamma_{i}, i>1\right\}$.

We denote $a\left(0, x^{\prime}, t\right) \sum^{\prime} c_{j} r_{j}$ by $h\left(0, x^{\prime}, t\right)$.

Remark 1. We can drop condition (c), since if $\left\{u_{\lambda}\right\}$ satisfies (a) and (b), then so does the sequence $\left\{g\left(x^{\prime}, t\right) u_{\lambda}\right\}$, where $g\left(x^{\prime}, t\right) \in C_{0}^{\infty}\left(\Gamma_{1} \times(0, T)\right)$ is a scalar function such that $g \equiv 1$ on $\operatorname{supp} a\left(0, x^{\prime}, t\right)$.

Remark 2. From the conclusion of Lemma 11.2, and the well-posed nature of the mixed problem, we also get $\left\|u_{\lambda}-e^{i \lambda \phi} h\right\|_{\Gamma_{1} \times[0, T]}=o(1)$ as $\lambda \rightarrow \infty$.

Remark 3. For convenience in proving Lemma 11.2, we may assume that $P_{1}$ is a constant matrix. This is easily attained by a change of dependent variable.

Remark 4. It suffices to prove a slightly weaker form of Lemma 11.2; namely, we can replace (b) by

(b') $\| P_{1}\left\{u_{\lambda}\left(0, x^{\prime}, t\right)-e^{i \lambda \phi\left(0, x^{\prime}, t\right)} h\left(0, x^{\prime}, t\right)\left\|\leqq o(1)+C_{1} \zeta\right\| h \|\right.$ where $\zeta$ is the diameter of the support of $h$.

We call this weaker form Lemma 11.2'.

Proof of Remark 4. Assume that Lemma 11.2' holds. Given, $h$ by using a Gårding-type partition of unity ([10]), we can write

$$
h=\sum_{j=1}^{N_{1}^{n}} h_{j} \equiv \sum \psi_{j}\left(x^{\prime}\right) h,
$$

where diameter $\sup \psi_{j}<c \zeta N_{0}^{-1}, \psi_{j} \in C_{0}^{\infty}\left(\Gamma_{1}\right), \sum \psi_{j}^{2}\left(x^{\prime}\right)=1$ if $x^{\prime} \in \operatorname{supp} h$ and where no point $x^{\prime}$ lies in the support of more than $n+1$ of the $\psi_{j}$ 's. According to Lemma 11.2', there exist $u_{\lambda}^{j}$ such that $u_{\lambda}^{j}$ satisfies (a), (c) and

$$
\left\|P_{1}\left(u_{\lambda}^{j}-e^{i \lambda \phi} h_{j}\right)\right\|^{2} \leqq o(1)+C \zeta^{2} N_{0}^{-2}\left\|\psi_{j} h\right\|^{2} .
$$

Thus, given $\varepsilon>0$, by first choosing $N_{0}$ and then $\lambda>0$ sufficiently large, we can attain

$$
\begin{gathered}
\left\|P_{1}\left(u_{\lambda}-e^{i \lambda \phi} h\right)\right\|^{2}=\left\|P_{1}\left(\sum u_{\lambda}^{j}-e^{i \lambda \phi} h\right)\right\|^{2} \\
\leqq \frac{\varepsilon}{2}\|h\|^{2}+\frac{\varepsilon}{2} \sum\left\|\psi_{j} h\right\|^{2}=\varepsilon\|h\|^{2}
\end{gathered}
$$

as well as

where $u_{\lambda}=\sum u_{\lambda}^{i}$.

$$
\left\|u_{\lambda}^{j}\right\|^{2}+\left\|L u_{\lambda}^{j}\right\|^{2} \leqq N_{0}^{-2 n} \varepsilon
$$

Consequently, $\left\{u_{\lambda}\right\}$ satisfies (a) and (b), and Remark 4 is proved.

Proof of Lemma 11.2'. Note first that $\sum^{\prime} c_{j} r_{j} \in C_{0}^{\infty}\left(R^{n}\right)$. For $X=(x, t, \eta, \tau)$ in a small conic neighborhood $V$ of $X_{0}=\left(0, x_{0}^{\prime}, t_{0}, \bar{\eta}, \bar{\tau}\right), M$ can be smoothly trans- 
formed to the block form

$$
M=q^{-1}\left[\begin{array}{cc}
M_{1} & 0 \\
0 & M_{-}
\end{array}\right] q .
$$

Here $M_{1}$ is a $k_{0} \times k_{0}$ block whose eigenvalues $\xi_{j k}$ satisfy $\xi_{j k}\left(X_{0}\right)=\bar{\xi}_{j}$, and

$$
\operatorname{Im} \xi_{j k}<-c \sqrt{\eta^{2}+\tau^{2}}, \quad c>0,
$$

uniformly for $X \in V$. We also require that at $X_{0}$, the eigenvalues of $M_{1} \operatorname{differ}$ from $\bar{\xi}_{j}$.

Let $L_{0}$ be the differential operator obtained by freezing the coefficients of $L$ at $\left(0, x_{0}^{\prime}, t_{0}\right)$, and let $M_{0}$ be defined as

$$
M_{0}=q_{0}^{-1}\left[\begin{array}{cc}
M_{1}^{0} & 0 \\
0 & M_{-}^{0}
\end{array}\right] q_{0}
$$

where $M_{0}, q_{0}, M_{1}^{0}$ and $M_{-}^{0}$ are obtained from $M, q, M_{1}$ and $M_{-}$, respectively, by freezing their coefficients at $\left(0, x_{0}^{\prime}, t_{0}\right)$. We shall denote the pseudo-differential operators in $\left(x^{\prime}, t\right)$, determined by $M, M_{0}, q_{0}, M_{1}^{0}$, and $M^{0}$, namely, $M\left(x, t ; D_{x^{\prime}}, D_{t}\right)$, $M_{0}\left(D_{x^{\prime}}, D_{t}\right)$, etc. , by $\mathscr{M}, \mathscr{M}_{0}, Q_{0}, \mathscr{M}_{1}^{0}$, and $\mathscr{M}^{0}$ respectively.

The symbol

$$
\hat{p}=q^{-1}\left[\begin{array}{cc}
I_{1} & 0 \\
0 & 0_{-}
\end{array}\right] q
$$

where $I_{1}$ is the identity operator on the domain of $M_{1}^{0}$ and $\mathcal{O}_{-}$is the zero operator on the domain of $M_{-}^{0}$, is a projection; that is, $\hat{p}^{2}=\hat{p}$. If we denote $\hat{p}\left(0, x_{0}^{\prime}, t_{0} ; \eta, \tau\right)$ by $p_{0}(\eta, \tau)$, then the operator $P_{0}$ defined by $P_{0}=p_{0}\left(D_{x^{\prime}}, D_{t}\right)$, is a projection operator. The following lemma will be helpful.

Lemma 11.3. $\operatorname{Lim}_{\lambda \rightarrow \infty}\left\|\left(1-P_{0}\right) e^{i \lambda(x \cdot \bar{\eta}+t \bar{\tau})} h\left(0, x^{\prime}, t\right)\right\|_{\Gamma_{1}} \leqq c \zeta\|h\|_{\Gamma_{1}}$, where $\zeta$ is the diameter of the support of $h$.

Proof of Lemma 11.3. By construction,

Therefore

$$
h\left(0, x^{\prime}, t\right)=\hat{p}\left(0, x^{\prime}, t ; \bar{\eta}, \bar{\tau}\right) h\left(0, x^{\prime}, t\right) \text {. }
$$

$$
\begin{aligned}
& \left\|\left(1-P_{0}\right) e^{i \lambda\left(x^{\prime} \cdot \bar{\eta}+t \bar{\tau}\right)} h\left(0, x^{\prime}, t\right)\right\|=\left\|\left(1-P_{0}\right) e^{i \lambda\left(x^{\prime} \cdot \bar{\eta}+t \bar{z}\right)} \hat{p}\left(0, x^{\prime}, t ; \bar{\eta}, \bar{\tau}\right) h\left(0, x^{\prime}, t\right)\right\| \\
& \quad \leqq \\
& \quad+\left\|\left(1-P_{0}\right) \hat{\rho}\left(0, x_{0}^{\prime}, t_{0} ; \bar{\eta}, \bar{\tau}\right) e^{i \lambda\left(x^{\prime} \cdot \bar{\eta}+t \bar{\tau}\right)} h\left(0, x^{\prime}, t\right)\right\| \\
& \quad \|\left(1-P_{0}\right)\left[\hat{p}\left(0, x^{\prime}, t ; \bar{\eta}, \bar{\tau}\right)-\hat{p}\left(0, x_{0}, t_{0} ; \bar{\eta}, \bar{\tau}\right] e^{i \lambda\left(x^{\prime} \cdot \eta+t \bar{\tau}\right)} h\left(0, x^{\prime}, t\right) \| .\right.
\end{aligned}
$$

Since $\left|\nabla_{x^{\prime}} \hat{p}\right|+\left|\nabla_{t} \hat{p}\right|$ is bounded, the second term on the right hand side of (11.6) can be estimated by $C \zeta\|h\|$. The first term tends to zero because the $\left(x^{\prime}, t\right)$ Fourier transform of $e^{i \lambda\left(x^{\prime} \cdot \bar{\eta}+t \bar{x}\right)} h\left(0, x^{\prime}, t\right)$ is concentrated in smaller and smaller conical neighborhoods of the ray in the direction of $(\bar{\eta}, \bar{\tau})$, and the symbol $\left(1-P_{0}\right)(\bar{\eta}, \bar{\tau})$. $\hat{p}\left(0, x_{0}^{\prime}, t_{0} ; \bar{\eta}, \bar{\tau}\right)$ equals zero. This proves the lemma.

Because of Lemma 11.3 and the concentrating effect of the multiplier $e^{i \lambda\left(x^{\prime} \cdot \eta+t \bar{\tau}\right)}$ on the conic support of $\tilde{h}$, we can replace $e^{i \lambda(x \cdot \bar{\eta}+t \bar{\tau})} h$ by $\psi\left(D_{x^{\prime}}, D_{t}\right)$. $e^{i \lambda\left(x^{\prime} \cdot \bar{\eta}+t \bar{t}\right)} h \equiv h_{\lambda}$, with error $o(1)$. Here $\psi^{\prime}\left(\xi^{\prime}, \tau\right)$ is a zero-order homogeneous 
symbol which is identically one in a small conic neighborhood of the ray $(c \bar{\eta}, c \bar{\tau}), c>0$, and is such that $\psi \equiv 0$ outside of a slightly larger neighborhood.

Consider now the solution $v_{\lambda}$ of the Cauchy problem

$$
\begin{array}{rlrl}
L_{0} v_{\lambda} & =0, & x_{1}>0 \\
v_{\lambda} & =h_{\lambda} \quad \text { on } \quad x_{1}=0 .
\end{array}
$$

Writing (11.7) in the form $\frac{\partial}{\partial x_{1}} v_{\lambda}=M v_{\lambda}$, we easily see from (11.4) and (11.5) that $v_{\lambda}$ satisfies an estimate of the form

$$
\left|\tilde{v}_{\lambda}\left(x_{1}, \eta, \tau\right)\right| \leqq c e^{-\frac{c}{2} \lambda x_{1}}\left|\tilde{h}_{\lambda}(\eta, \tau)\right| .
$$

This implies the global estimate

$$
\left\|v_{\lambda}\right\| \leqq c \lambda^{-\frac{1}{2}} \text {. }
$$

Now let $u_{\lambda}$ be the solution of the mixed problem (cf. KREISs [17])

$$
\begin{array}{ll}
\text { (a) } L u_{\lambda}=0, & x_{1}>0, t>0 \\
\text { (b) } P u_{\lambda}=P v_{\lambda}, & x_{1}=0 \\
\text { (c) } u_{\lambda}=v_{\lambda} & t<0, x_{1}>0 .
\end{array}
$$

Then the difference $w_{\lambda}=u_{\lambda}-v_{\lambda}$ satisfies

For fixed $x_{1}$,

$$
\begin{aligned}
L w_{\lambda} & =-L v_{\lambda}=\left(L_{0}-L\right) v_{\lambda}, \quad x_{1}>0, t>0 \\
P w_{\lambda} & =0, \quad x_{1}=0 \\
w_{\lambda} & =0, \quad t<0 .
\end{aligned}
$$

$$
\left\|\left(L_{0}-L\right) v_{\lambda}\right\| \leqq c \sum_{j=1}^{n} \frac{\partial}{\partial x_{j}}\left\|v_{\lambda}\right\| \leqq c^{\prime} e^{-\frac{\lambda}{2} x_{1}}(\lambda+1)\left\|h_{\lambda}\right\|_{1} .
$$

Hence $\left\|L\left(u_{\lambda}-v_{\lambda}\right)\right\|=o(1)$ as $\lambda \rightarrow \infty$. From the assumed well-posed nature of the mixed problem, we then have $\left\|u_{\lambda}-v_{\lambda}\right\|=o(1)$ as $\lambda \rightarrow \infty$, and hence also $\left\|u_{\lambda}\right\|=o(1)$ as $\lambda \rightarrow \infty$. Thus $\left\{u_{\lambda}\right\}$ has the properties required for the conclusion of Lemma 11.2', namely (a) and $\left(b^{\prime}\right)$.

Remark. The results of this section, together with the obvious stability of the counterexample in $\$ 9$ under small perturbations of the coefficients, show that if we allow the coefficients and boundary conditions to vary smoothly, the counterexample persists.

Sponsored by the United States Army under Contract No. DA-31-124-ARO-D-462, at Mathematics Research Center, University of Wisconsin, Madison.

\section{References}

1. AGMON, S., Problèmes mixtes pour les équations hyperboliques d'ordre supéreure. Colloques Internationales du Centre National de la Récherche Scientifique, 117, Paris 1962.

2. AGEMI, R., On energy inequalities of mixed problems for hyperbolic equations of second order. J. Fac. Science, Hokkaido Univ. (1) 21, 221-236 (1971). 
3. Agranovich, M.S., Theorem on matrices depending on parameters and its applications to hyperbolic systems. Funktstional'nyi Analiz i ego Prilozheniya 6, 1-11 (1972).

4. BLOoM, C. O., On the validity of the geometrical theory of diffraction by starshaped cylinders. J. Math. Anal. and Appl. 40, 107-121 (1972).

5. Borovikov, V. A., The elementary solution of partial differential equations with constant coefficients. Trudy Moskov. Mat. Obšč. 68, 159-257 (1959).

6. BurRIDGe, R., Lacunas in two-dimensional wave propagation. Proc. Cambridge Phil. Soc. 63, 819-825 (1967).

7. Courant, R., \& D. Hilbert, Methods of Mathematical Physics, Vol. II, Partial Differential Equations. New York: Interscience Publishers 1962.

8. Duff, G. F. D., On wave fronts and boundary waves. Comm. Pure Appl. Math. 17, 189-225 (1964).

9. Duistermaat, J. J., \& L. Hörmander, Fourier integral operators II. Acta Math. 128, 183-269 (1972).

10. GärdING, L., Solution directe du problème de Cauchy pour les équations hyperboliques. Proc. Coll. Int. du C.N.R.S. 71, 71-90 (1956).

11. Hersh, R., Mixed problems in several variables. J. Math. Mech. 12, 317-334 (1963).

12. HöRMANDER, L., Fourier integral operators, I. Acta Mathematica 127, 79-183 (1971).

13. IKaWA, M., Mixed problems for hyperbolic equations of second order. J. Math. Soc. Japan 20, 580-608 (1968).

14. KelleR, J. B., Geometrical theory of diffraction. J. Opt. Soc. Amer. 52, 116-130 (1962).

15. KUPKA, I. A. K., \& S. OsHER, On the wave equation in a multi-dimensional corner. Comm. Pure Appl. Math. 24, 381-394 (1971).

16. KAIITANI, K., Initial-boundary value problems for first order hyperbolic systems. R.I.M.S., Kyoto Univ. 7, 181-204 (1971/72).

17. KreIss, H.-O., Initial boundary value problems for first order hyperbolic systems. Comm. Pure Appl. Math. 23, 277-298 (1970).

18. LAx, P. D., Asymptotic solutions of oscillatory initial value problems. Duke Math. J. 24, 627-646 (1957).

19. LAx, P. D., \& R. S. PhillIPs, Scattering Theory. New York: Academic Press 1967.

20. LudwiG, D., Exact and asymptotic solutions of the Cauchy problem. Comm. Pure Appl. Math. 13, 473-508 (1960).

21. Ludwig, D., \& C. S. Morawetz, An inequality for the reduced wave operator and the justification of geometrical optics. Comm. Pure Appl. Math. 21, 187-203 (1968).

22. Nirenberg, L., Lectures on Linear Partial Differential Equations, Conference Board of the Mathematical Sciences, Regional Conference Series in Mathematics \# 17.

23. OsHer, S., Initial-boundary value problems for hyperbolic systems in regions with corners, I. Trans. Amer. Math. Soc. 176, 141-164 (1973).

24. Osher, S., An ill posed problem for a hyperbolic equation near a corner. Bull. Amer. Math. Soc. 79, 1043-1044 (1973).

25. Ralston, J. V., Note on a paper of Kreiss. Comm. Pure Appl. Math. 24, 759-762 (1971).

26. Ralston, J. V., Solutions of the wave equation with localized energy. Comm. Pure Appl. Math. 22, 807-823 (1969).

27. RAUCH, J., $\mathscr{L}_{2}$ is a continuable initial condition for Kreiss' mixed problem. Comm. Pure Appl. Math. 25, 265-285 (1972).

28. RAUCH, J., Energy and resolvent inequalities for hyperbolic mixed problems. J. Diff. Equations 11, 528-540 (1972).

29. RAUCH, J., \& F. J. MASSEY, Differentiability of solutions to hyperbolic initial-boundary value problems. Trans. Amer. Math. Soc. 189, 303-318 (1974).

30. Sadamatsu, $T$., On mixed problems for hyperbolic systems of first order with constant coefficients. J. Math. of Kyoto Univ. 9, 339-361 (1969).

31. SAKAMOto, R., Mixed problems for hyperbolic equations, II. J. Math. of Kyoto Univ. 10, 403-417 (1970).

32. Sarason, L., On hyperbolic mixed problems. Arch. Rational Mech. Anal. 18, 311-334 (1965).

33. Sarason, L., Elliptic regularization for symmetric positive systems. J. Math. Mech. 16, 807-827 (1967). 
34. Sarason, L., Symmetrizable systems in regions with corners and edges. J. Math. Mech. 19, 601-607 (1970).

35. Sarason, L., On weak and strong solutions of boundary value problems. Comm. Pure Appl. Math., 15, 237-288 (1962).

36. Shirota, T., \& K. Asano, On mixed problems for regularly hyperbolic systems. J. of the Faculty of Science, Kokkaido Univ. (1) 21, 1-45 (1970).

37. SHIRota, T., \& R. AGEMi, On certain mixed problems for hyperbolic equations of higher order, III. Proc. Japan Acad. 45, 854-858 (1969).

38. SHIROTA, T., \& R. AGEMi, On necessary and sufficient conditions for $L^{2}$-well posedness of mixed problems for hyperbolic equations. J. Faculty of Science, Hokkaido Univ. (1) 21, 133-151 (1970).

39. StRANG, G., On strong hyperbolicity. J. Math. Kyoto Univ. 6, 397-417 (1967).

40. Strang, G., Hyperbolic initial-boundary value problems in two unknown. J. Diff. Equations 6, 161-171 (1969).

41. ATIYAH, M. F., R. BotT, \& L. GÅRDING, Lacunas for hyperbolic differential operators with constant coefficients. Acta Math. 126, 109-189 (1970).

42. Petrovsky, I. G., On the diffusion of waves and the lacunas for hyperbolic equations. Mat. $86,17(59), 289-370$ (1945).

\section{Department of Mathematics University of Washington Seattle \\ and \\ Department of Mathematics University of Michigan Ann Arbor}

(Received July 5, 1973) 